\title{
Strong approximation and descent
}

\author{
By Ulrich Derenthal at Hannover and Dasheng Wei at Beijing
}

\begin{abstract}
We introduce descent methods to the study of strong approximation on algebraic varieties. We apply them to two classes of varieties defined by $P(\mathbf{t})=N_{K / k}(\mathbf{z})$ : firstly for quartic extensions of number fields $K / k$ and quadratic polynomials $P(\mathbf{t})$ in one variable, and secondly for $k=\mathbb{Q}$, an arbitrary number field $K$ and $P(\mathbf{t})$ a product of linear polynomials over $\mathbb{Q}$ in at least two variables. Finally, we illustrate that a certain unboundedness condition at archimedean places is necessary for strong approximation.
\end{abstract}

\section{Introduction}

Let $k$ be a number field. We study strong approximation with Brauer-Manin obstruction for two families of algebraic varieties $X \subset \mathbb{A}_{k}^{n+s}$ defined by equations of the form

$$
P(\mathbf{t})=N_{K / k}(\mathbf{z}),
$$

where $P(\mathbf{t}) \in k\left[t_{1}, \ldots, t_{s}\right]$ is a polynomial in $s$ variables over $k$ and $N_{K / k}$ is a norm form for an extension $K / k$ of degree $n$.

In our proofs, we use descent to reduce the problem to strong approximation on their universal torsors. While descent has been applied frequently to weak approximation, the precise formulation of our descent lemma seems to be crucial for its first applications to strong approximation.

To prove strong approximation on universal torsors, we reduce it to quadrics by the fibration method in one case, and we use a generalization of a result of Browning and Matthiesen, based on methods of Green, Tao, and Ziegler from additive combinatorics, in the other case.

In our results, we encounter an unboundedness condition at the archimedean places. We give a counterexample to strong approximation that shows that such a condition is generally necessary.

Background. Let $X$ be an algebraic variety defined over a number field $k$. We say that strong approximation holds for $X$ off a finite set $S$ of places of $k$ if the image of the set $X(k)$ of rational points on $X$ is dense in the space $X\left(\mathbb{A}_{k}^{S}\right)$ of adelic points on $X$ outside $S$. Strong

The first author was supported by the Deutsche Forschungsgemeinschaft (Grant No. DE 1646/2-1 and DE 1646/3-1). The second author is supported by National Key Basic Research Program of China (Grant No. 2013CB834202) and National Natural Science Foundation of China (Grant No. 11371210 and 11321101). 
approximation for $X$ off $S$ implies the Hasse principle for $S$-integral points on any $S$-integral model of $X$.

For a proper variety $X$, strong approximation off $S=\emptyset$ is the same as weak approximation. For an affine variety $X$, however, studying strong approximation and the Hasse principle for integral points on integral models seems to be generally much harder than studying weak approximation and the Hasse principle for rational points on proper models of $X$.

Failures of weak approximation and the Hasse principle for rational points on proper varieties are often explained by Brauer-Manin obstructions, introduced by Manin [26]. Only recently this was generalized to strong approximation by Colliot-Thélène and $\mathrm{Xu}$ [14].

For algebraic groups and their homogeneous spaces, weak and strong approximation and the Hasse principle have been widely studied. For certain simply connected semisimple groups and their principal homogeneous spaces, we have the classical work of Kneser, Harder, Platonov and others. For certain homogeneous spaces of connected algebraic groups with connected or abelian stabilizers, see for example $[1,2,14,16,20,32,33]$ and the references therein for weak and strong approximation with Brauer-Manin obstruction. This includes varieties defined by equation (1.1) for constant $P$ and arbitrary $K / k$, and for $s=1$ with $\operatorname{deg}(P(t))=2$ and $[K: k]=2$.

Much less is known for more general varieties that are not homogeneous spaces of algebraic groups. For weak approximation with Brauer-Manin obstruction, let us mention the now classical example of Châtelet surfaces [10,11], which are actually smooth proper models of certain varieties defined by $(1.1)$. See $[3,17,30]$ and the references therein for weak approximation results for several further classes of varieties defined by (1.1).

A recent breakthrough is the introduction of deep results from additive combinatorics due to Green-Tao-Ziegler and Matthiesen to deduce weak approximation for varieties of the form $(1.1)$ when $P(t)$ is a product of arbitrarily many linear polynomials over $\mathbb{Q}([4,5,21])$.

For strong approximation with Brauer-Manin obstruction for affine varieties defined by equations of the form

$$
P(t)=q\left(z_{1}, z_{2}, z_{3}\right),
$$

see [15]. For more general fibrations over $\mathbb{A}_{k}^{1}$ with split (e.g., geometrically integral) fibers, see [8].

Colliot-Thélène and Harari $[8$, p.4] ask for the integral Hasse principle and strong approximation for equation (1.1) with $s=1$, a separable polynomial $P(t)$ of degree at least 3 , and $[K: k]=2$. They say that this is out of reach of the current techniques because of the following two essential difficulties: on the one hand, $\operatorname{Br}\left(X_{t}\right) / \operatorname{Br}(k)$ is infinite for each smooth $k$-fiber $X_{t}$ for the natural fibration $X \rightarrow \mathbb{A}_{k}^{1}$ via projection to the $t$-coordinate, and on the other hand, the fibers over the roots of $P(t)$ are not split. Note that the same difficulties occur for $P(t)$ of degree at least 2 , and $[K: k] \geq 3$.

Counterexamples to strong approximation explained by Brauer-Manin obstructions can be found in [18,25]. See also [13] for computations of Brauer-Manin obstructions for integral points on certain cubic surfaces.

Our results. We obtain the first strong approximation results for varieties defined by equation (1.1). Here, both of the difficulties occur that were mentioned in [8, p. 4]. In our main theorems, we consider two families of such varieties. Our notation is mostly standard; see the end of the introduction for a reminder. 
Theorem 1.1. Let $K / k$ be an extension of number fields. Assume that

$$
P(t)=c\left(t^{2}-a\right) \in k[t]
$$

is an irreducible quadratic polynomial, and $[K: k]=4$ with $\sqrt{a} \in K$. Let $X \subset \mathbb{A}_{k}^{5}$ be defined by (1.1) with $s=1$. Assume that there is an archimedean place $v_{0}$ such that $p\left(X\left(k_{v_{0}}\right)\right)$ is not bounded, where $p: X \rightarrow \mathbb{A}_{k}^{1}$ is the projection to the $t$-coordinate. Then strong approximation with Brauer-Manin obstruction holds for $X$ off $v_{0}$.

We also compute $\operatorname{Br}(X)$ for $X$ as in Theorem 1.1, and in certain cases we deduce the Hasse principle for integral points and strong approximation (without Brauer-Manin obstructions), see Corollaries 4.5 and 4.6.

Theorem 1.2. Let $k=\mathbb{Q}$. For $s \geq 2$, let $P(\mathbf{t}) \in \mathbb{Q}\left[t_{1}, \ldots, t_{s}\right]$ be a product of pairwise proportional or affinely independent linear polynomials over $\mathbb{Q}$. Let $K / \mathbb{Q}$ be an arbitrary extension of number fields of degree $n$. Let $X \subset \mathbb{A}_{\mathbb{Q}}^{n+s}$ be the affine variety defined by (1.1). Let $C$ be the union of the connected components of $p\left(X^{\mathrm{sm}}(\mathbb{R})\right)$ that contain balls of arbitrarily large radius, where $p: X \rightarrow \mathbb{A}_{k}^{s}$ is the projection to the $\mathbf{t}$-coordinates. Then $X^{\mathrm{sm}}(k)$ is dense in the image of $\left(p^{-1}(C) \times X^{\mathrm{sm}}\left(\mathbb{A}_{k}^{f}\right)\right)^{\mathrm{Br}_{1}\left(X^{\mathrm{sm}}\right)}$ in $X\left(\mathbb{A}_{k}^{f}\right)$. In particular, if $K$ is not totally imaginary or if the factors of $P(\mathbf{t})$ are linear forms, then $C=p\left(X^{\mathrm{sm}}(\mathbb{R})\right)$, and $X$ satisfies smooth strong approximation with algebraic Brauer-Manin obstruction off $\infty$ (see Definition 2.1).

Furthermore, we can deduce a smooth Hasse principle with Brauer-Manin obstruction for integral points on $X$ as in Theorem 1.2. See Corollary 5.7 for details.

Techniques. There are two fundamental techniques to reduce the study of weak approximation and the Hasse principle for rational points (possibly with Brauer-Manin obstruction) for one class of varieties to the same questions for other varieties, namely the fibration method and the descent method. One may ask whether these techniques can also be applied in the context of strong approximation.

The fibration method typically applies to fibrations $f: X \rightarrow Y$ where weak approximation or the Hasse principle is known both for the fibers of $f$ and the base $Y$. An example is the deduction of the Hasse principle for quadratic forms in five variables from the fourvariable-case. See [10,11] for more involved applications, and [19] for its combination with Brauer-Manin obstructions.

Generalizing the fibration method to strong approximation is achieved in some generality in $[8,15]$.

The descent method reduces the study of weak approximation and the Hasse principle with (algebraic) Brauer-Manin obstruction to their study on torsors under tori over the original variety (for example universal torsors). This is expected to simplify the task since typically no (algebraic) Brauer-Manin obstruction occurs on universal torsors. See [7, 10,11] for applications of descent to weak approximation.

To our knowledge, Theorems 1.1 and 1.2 are the first applications of the descent method to strong approximation. For this, an important auxiliary result is the descent lemma presented in Section 3. A subtle point was to find the right formulation that makes it applicable in practice. Then the proof of this lemma is an easy application of descent theory as introduced by Colliot-Thélène and Sansuc, see [9]. 
Our descent lemma reduces strong approximation with algebraic Brauer-Manin obstruction on the original variety to strong approximation on auxiliary varieties containing open subsets of universal torsors.

For $X$ as in Theorem 1.1, we have determined a local description of universal torsors in [17]. Here, we observe that these are essentially fibrations over $\mathbb{A}_{k}^{4}$ whose fibers are smooth 3-dimensional quadrics, so that an application of the fibration method yields the result.

For $X$ as in Theorem 1.2, we show that the universal torsors are essentially varieties for which weak approximation was proved in recent work of Browning and Matthiesen [4] (on $X$ defined over $k=\mathbb{Q}$ by (1.1) in cases where $P(t)$ is totally split over $\mathbb{Q}$, with $s=1$; note that we cannot treat the case $s=1$ in Theorem 1.2, see Remark 5.5 for the reason), based on results from additive combinatorics by Green-Tao-Ziegler and Matthiesen. From a generalization of the key technical result [4, Theorem 5.2] of Browning and Matthiesen from linear forms to linear polynomials, we deduce that the varieties containing our universal torsors also satisfy strong approximation.

The descent method may lead to further results on strong approximation for varieties defined by (1.1) when combined with other analytic techniques. For example, it would be interesting to combine it with sieve methods (see [17,22,23] for results on weak approximation) and the circle method (see [22] and [28] for results on weak approximation).

An unboundedness condition. We observe that Theorem 1.1 and Theorem 1.2 include unboundedness conditions at an archimedean place. This has some resemblance with conditions at archimedean places appearing in strong approximation results on homogeneous spaces of algebraic groups, e.g., [27, Theorem 7.12].

Example 6.2 shows that strong approximation with Brauer-Manin obstruction off $\infty$ does not hold for the variety $X \subset \mathbb{A}_{\mathbb{Q}}^{3}$ defined by

$$
t(t-2)(t-10)=x^{2}+y^{2},
$$

which is an example of equation (1.1). Here, $X(\mathbb{R})$ has a bounded and an unbounded connected component.

This counterexample and Theorems 1.1-1.2 lead us to the expectation that only the following version of smooth strong approximation can be true for varieties defined by (1.1). See also [8].

Question 1.3. Let $K / k$ be an extension of number fields of degree $n$, let $P(\mathbf{t})$ be a non-constant polynomial over $k$ in $s$ variables. Let $X \subset \mathbb{A}_{k}^{n+s}$ be the affine variety over $k$ defined by (1.1). Let $v_{0}$ be an archimedean place. Let $p: X \rightarrow \mathbb{A}_{k}^{s}$ be the projection to the t-coordinates. Let $C$ be the union of the connected components of $p\left(X^{\mathrm{sm}}\left(k_{v_{0}}\right)\right)$ that contain balls of arbitrarily large radius. Is $X^{\mathrm{sm}}(k)$ dense in the image of $\left(p^{-1}(C) \times X^{\mathrm{sm}}\left(\mathbb{A}_{k}^{\left\{v_{0}\right\}}\right)\right)^{\operatorname{Br}\left(X^{\mathrm{sm}}\right)}$ in $\left.X\left(\mathbb{A}_{k}^{\left\{v_{0}\right\}}\right)\right)$ with respect to the adelic topology?

Theorems 1.1 and 1.2 give an affirmative answer to this question for two families of varieties.

Terminology. For a field $k$ of characteristic 0 , fix an algebraic closure $\bar{k}$, and let $\Gamma_{k}$ be the absolute Galois group. Let $\operatorname{Br}(k)$ be the Brauer group of $k$. For a scheme $X$ over $k$, let $X^{\text {sm }}$ 
be its smooth locus, and let $\bar{X}:=X \times_{k} \bar{k}$. Let $\operatorname{Br}(X):=H_{\text {êt }}^{2}\left(X, \mathbb{G}_{\mathrm{m}}\right)$ be the cohomological Brauer group, $\operatorname{Br}_{0}(X)$ its subgroup of constant elements, namely the image of the natural map $\operatorname{Br}(k) \rightarrow \operatorname{Br}(X)$, and $\operatorname{Br}_{1}(X)$ its algebraic Brauer group, namely the kernel of the natural map $\operatorname{Br}(X) \rightarrow \operatorname{Br}(\bar{X})$.

Now let $k$ be a number field. Then $\Omega_{k}$ denotes the set of places of $k$, and $\infty_{k}$ denotes its subset of archimedean places. We write $v<\infty_{k}$ for $v \in \Omega_{k} \backslash \infty_{k}$. The ring of integers in $k$ is denoted by $\mathcal{O}_{k}$. For $v \in \Omega_{k}$, let $k_{v}$ be the completion of $k$ at the place $v$, and let, for $v<\infty_{k}$, $\mathcal{O}_{v}$ be the ring of integers in $k_{v}$. The adele ring with its usual adelic topology is denoted by $\mathbb{A}_{k}$. For a finite subset $S \subset \Omega_{k}$, let $\mathcal{O}_{S}$ be the ring of $S$-integers of $k$, and let $\mathbb{A}_{k}^{S} \subset \prod_{v \in \Omega_{k} \backslash S} k_{v}$ be the adeles without $v$-component for all $v \in S$, which also comes with a natural adelic topology. In particular, we write $\mathbb{A}_{k}^{f}:=\mathbb{A}_{k}^{\infty_{k}}$ for the adeles without archimedean components.

Let $X$ be a geometrically integral variety over a number field $k$, and let $S \subset \Omega_{k}$ be a finite set of places. We say that strong approximation holds for $X$ off $S$ if $X(k)$ is dense in the image of $X\left(\mathbb{A}_{k}\right)$ in $X\left(\mathbb{A}_{k}^{S}\right)$.

One says that strong approximation with (algebraic) Brauer-Manin obstruction holds for $X$ off $S$ if $X(k)$ is dense in the image of $X\left(\mathbb{A}_{k}\right)^{\operatorname{Br}(X)}\left(\operatorname{resp} . X\left(\mathbb{A}_{k}\right)^{\mathrm{Br}_{1}(X)}\right)$ in $X\left(\mathbb{A}_{k}^{S}\right)$ under the natural projection (see [15, Definition 2.4]). Here, $X\left(\mathbb{A}_{k}\right)^{B}$ is the set of all adelic points $\left(x_{v}\right) \in X\left(\mathbb{A}_{k}\right)$ satisfying

$$
\sum_{v \in \Omega_{k}} \operatorname{inv}_{v}\left(\beta\left(x_{v}\right)\right)=0
$$

for all $\beta$ in a subset $B \subset \operatorname{Br}(X)$, where $\operatorname{inv}_{v}: \operatorname{Br}\left(k_{v}\right) \rightarrow \mathbb{Q} / \mathbb{Z}$ is the invariant map from local class field theory.

Acknowledgement. We thank J.-L. Colliot-Thélène and L. Matthiesen for useful remarks and discussions, in particular during the first author's stay at the Institut de Hautes Études Scientifiques (September/October 2013), whose support and hospitality is gratefully acknowledged. We are grateful to the anonymous referees for their helpful comments.

\section{Strong approximation on singular varieties}

Let $k$ be a number field. For smooth varieties $X$ over $k$, it is interesting to study strong approximation because we can derive the existence of integral points on any integral model of $X$.

For singular varieties $X$ over $k$, the implicit function theorem may fail, and hence we cannot hope to prove strong approximation on $X$. On the other hand, integral models of $X^{\text {sm }}$ often have far less integral points than integral models of $X$, hence strong approximation on $X^{\text {sm }}$ is generally too much to ask for.

Instead, we introduce the following notion of smooth strong approximation on $X$ which is suitable to determine the existence of integral points on any integral model of the singular variety $X$ (see Remark 2.2 and Corollary 5.7).

Definition 2.1. Let $X$ be a geometrically integral variety over a number field $k$. Let $S$ be a finite set of places of $k$. We say that $X$ satisfies smooth strong approximation off $S$ if $X^{\mathrm{sm}}(k)$ is dense in the image of $X^{\mathrm{sm}}\left(\mathbb{A}_{k}\right)$ in $X\left(\mathbb{A}_{k}^{S}\right)$ under the natural projection. 
Analogously, we say that $X$ satisfies smooth strong approximation with (algebraic) Brauer-Manin obstruction off $S$ if $X^{\mathrm{sm}}(k)$ is dense in the image of $X^{\mathrm{sm}}\left(\mathbb{A}_{k}\right)^{\operatorname{Br}\left(X^{\mathrm{sm}}\right)}$ (resp. in the image of $\left.X^{\mathrm{sm}}\left(\mathbb{A}_{k}\right)^{\mathrm{Br}_{1}\left(X^{\mathrm{sm}}\right)}\right)$ in $X\left(\mathbb{A}_{k}^{S}\right)$.

We say that $X$ satisfies the smooth integral Hasse principle (with (algebraic) BrauerManin obstruction) if the following holds for any integral model $\mathfrak{X}$ of $X$ : If

$$
X^{\mathrm{sm}}\left(\mathbb{A}_{k}\right)^{B} \cap\left(\prod_{v \in \infty_{k}} X\left(k_{v}\right) \times \prod_{v<\infty_{k}} \mathfrak{X}\left(\mathcal{O}_{v}\right)\right)
$$

is non-empty (where $B$ is $\emptyset$ resp. $\operatorname{Br}\left(X^{\mathrm{sm}}\right)$ resp. $\operatorname{Br}_{1}\left(X^{\mathrm{sm}}\right)$ ), then there are integral points on $\mathcal{X}$.

For singular $X$, note that smooth strong approximation on $X$ off $S$ is not the same as strong approximation on $X^{\mathrm{sm}}$ : the latter means that $X^{\mathrm{sm}}(k)$ is dense in the image of $X^{\mathrm{sm}}\left(\mathbb{A}_{k}\right)$ in $X^{\mathrm{sm}}\left(\mathbb{A}_{k}^{S}\right)$, whose adelic topology is stronger than the topology induced by $X\left(\mathbb{A}_{k}^{S}\right)$.

Remark 2.2. Assume that $X$ is a variety over a number field $k$ satisfying smooth strong approximation (with (algebraic) Brauer-Manin obstruction) off $\infty_{k}$. Then the smooth integral Hasse principle (with (algebraic) Brauer-Manin obstruction) holds on any integral model of $X$.

Indeed, for any integral model $\mathfrak{X}$, we have $\left(q_{v}\right)$ in the set (2.1). An open neighborhood of $\left(q_{v}\right)_{v<\infty_{k}} \in X\left(\mathbb{A}_{k}^{f}\right)$ is given by $\prod_{v<\infty_{k}} \mathfrak{X}\left(\mathcal{O}_{v}\right)$. Then smooth strong approximation off $\infty_{k}$ gives a $q \in X^{\mathrm{sm}}(k)$ which lies in this open neighborhood. This ensures $q \in \mathfrak{X}\left(\mathcal{O}_{k}\right)$.

Next, we compare smooth strong approximation to central strong approximation (see [15, Section 8]). Recall that it can be defined as follows:

Definition 2.3 (Colliot-Thélène, $\mathrm{Xu}$ ). Let $X$ be a geometrically integral variety over a number field $k$. Let $S$ be a finite set of places of $k$. Let $f: \widetilde{X} \rightarrow X$ be a resolution of singularities for $X$. The following two definitions do not depend on the choice of $\widetilde{X}$.

One says that central strong approximation holds for $X$ off $S$ if the diagonal image of $X^{\mathrm{sm}}(k)$ is dense in the natural image of $\widetilde{X}\left(\mathbb{A}_{k}\right)$ in $X\left(\mathbb{A}_{k}^{S}\right)$.

Assume that $\operatorname{Br}(\widetilde{X}) / \operatorname{Br}_{0}(\widetilde{X})\left(\operatorname{resp} . \operatorname{Br}_{1}(\widetilde{X}) / \operatorname{Br}_{0}(\widetilde{X})\right)$ is finite. One says that central strong approximation with (algebraic) Brauer-Manin obstruction holds for $X$ off $S$ if the diagonal image of $X^{\mathrm{sm}}(k)$ is dense in the natural image of $\widetilde{X}\left(\mathbb{A}_{k}\right)^{\operatorname{Br}(\widetilde{X})}\left(\right.$ resp. $\left.\widetilde{X}\left(\mathbb{A}_{k}\right)^{\mathrm{Br}_{1}(\widetilde{X})}\right)$ in $X\left(\mathbb{A}_{k}^{S}\right)$.

Note that the finiteness assumption on the Brauer group is generally necessary to ensure that the definition of central strong approximation with Brauer-Manin obstruction is independent of the choice of $\widetilde{X}$, but that no such finiteness condition is needed in our definition of smooth strong approximation.

Remark 2.4. Let $X$ be a geometrically integral variety over a number field $k$, and let $S$ be a finite set of places of $k$. Then smooth strong approximation on $X$ off $S$ is equivalent to central strong approximation on $X$ off $S$.

Assume that the quotient $\operatorname{Br}\left(X^{\mathrm{sm}}\right) / \mathrm{Br}_{0}\left(X^{\mathrm{sm}}\right)\left(\operatorname{resp} . \mathrm{Br}_{1}\left(X^{\mathrm{sm}}\right) / \mathrm{Br}_{0}\left(X^{\mathrm{sm}}\right)\right)$ is finite. Then smooth strong approximation with (algebraic) Brauer-Manin obstruction on $X$ off $S$ is equivalent to central strong approximation with (algebraic) Brauer-Manin obstruction on $X$ off $S$.

This equivalence is proved via the version [6, Théorème 1.4] of Harari's formal lemma [19, Corollaire 2.6.1]. 


\section{A descent lemma}

The following lemma, based on descent theory of Colliot-Thélène and Sansuc [9], is central in our proofs of strong approximation in the following sections.

Lemma 3.1. Let $k$ be a number field. Let $X$ be an integral variety over $k$ with $\operatorname{Pic}\left(\bar{X}^{\mathrm{sm}}\right)$ of finite type and $\bar{k}\left[X^{\mathrm{sm}}\right]^{\times}=\bar{k}^{\times}$, and let $U$ be a dense open subset of $X^{\mathrm{sm}}$. Let $S$ be a finite subset of $\Omega_{k}$. For any universal torsor $f: \mathcal{T} \rightarrow X^{\mathrm{sm}}$, assume that its restriction $\mathcal{T}_{U}:=\mathcal{T} \times_{X^{\mathrm{sm}}} U$ is geometrically integral, and that there is a commutative diagram

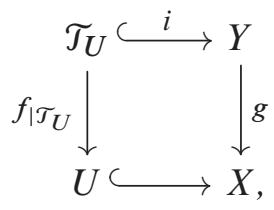

where $Y$ is a variety over $k$ satisfying smooth strong approximation off $S$, and $i: \mathcal{T}_{U} \rightarrow Y$ is an open immersion. Then $X$ satisfies smooth strong approximation with algebraic Brauer-Manin obstruction off $S$.

Proof. We must find a rational point $p \in X^{\mathrm{sm}}(k)$ which approximates the projection of a given $\left(p_{v}\right) \in X^{\mathrm{sm}}\left(\mathbb{A}_{k}\right)^{\mathrm{Br}_{1}\left(X^{\mathrm{sm}}\right)}$ in $X\left(\mathbb{A}_{k}^{S}\right)$.

By descent theory (see [9], [29, Theorem 3]), there is a universal torsor $f: \mathcal{T} \rightarrow X^{\mathrm{sm}}$ and $\left(r_{v}\right) \in \mathcal{T}\left(\mathbb{A}_{k}\right)$ such that $\left(f\left(r_{v}\right)\right)=\left(p_{v}\right)$.

Since $\mathcal{T}_{U}$ is geometrically integral, any integral model $\mathfrak{\Upsilon}_{U}$ of $\mathcal{T}_{U}$ satisfies $\mathfrak{\Upsilon}_{U}\left(\mathcal{O}_{v}\right) \neq \varnothing$ for almost all $v<\infty_{k}$. This implies that $\mathcal{T}_{U}\left(\mathbb{A}_{k}\right)$ is dense in $\mathcal{T}\left(\mathbb{A}_{k}\right)$ since $\mathcal{T}$ is smooth and $\mathcal{T}_{U}$ is open and dense in it. Therefore, we can find $\left(r_{v}^{\prime}\right) \in \mathcal{T}_{U}\left(\mathbb{A}_{k}\right)$ very close to $\left(r_{v}\right)$ in $\mathcal{T}\left(\mathbb{A}_{k}\right)$.

By assumption, we have

$$
\mathcal{T}_{U} \subset Y^{\mathrm{sm}} \subset Y
$$

for some $Y$ satisfying smooth strong approximation off $S$. Hence we obtain a point $r \in Y^{\mathrm{sm}}(k)$ arbitrarily close to the projection of $\left(r_{v}^{\prime}\right) \in \mathcal{T}_{U}\left(\mathbb{A}_{k}\right) \subset Y^{\mathrm{sm}}\left(\mathbb{A}_{k}\right)$ in $Y\left(\mathbb{A}_{k}^{S}\right)$; for $r$ close enough, we have $r \in \mathcal{T}_{U}(k)$.

Let $p:=f(r) \in U(k)$. Since $\left(r_{v}^{\prime}\right)$ is very close to $\left(r_{v}\right)$ in $\mathcal{T}\left(\mathbb{A}_{k}\right)$, we know that the point $\left(p_{v}^{\prime}\right):=\left(f\left(r_{v}^{\prime}\right)\right)$ is very close to $\left(p_{v}\right)$ in $X^{\mathrm{sm}}\left(\mathbb{A}_{k}\right)$. Since $X^{\mathrm{sm}}$ is open in $X$, also $\left(p_{v}^{\prime}\right)$ is very close to $\left(p_{v}\right)$ with respect to the adelic topology of $X\left(\mathbb{A}_{k}\right)$. Furthermore, since $r$ is very close to the projection of $\left(r_{v}^{\prime}\right)$ in $Y\left(\mathbb{A}_{k}^{S}\right)$, we have $p$ very close to the projection of $\left(p_{v}^{\prime}\right)=\left(g\left(r_{v}^{\prime}\right)\right)$ in $X\left(\mathbb{A}_{k}^{S}\right)$. Hence $p$ is very close to the projection of $\left(p_{v}\right)$ in $X\left(\mathbb{A}_{k}^{S}\right)$.

\section{Quadratic polynomials represented by quartic norms}

In this section, we apply our Descent Lemma 3.1 to prove Theorem 1.1. The main work lies in Proposition 4.1 proving strong approximation for the varieties $Y \subset \mathbb{A}_{k}^{8}$ defined by (4.2), which essentially are the universal torsors, using their fibration over $\mathbb{A}_{k}^{4}$ whose fibers are threedimensional quadrics.

Additionally, we compute the Brauer group of $X$ in Proposition 4.4 and obtain results on the integral Hasse principle. 
Proof of Theorem 1.1. Let $X$ be as in Theorem 1.1. Since $P(t)$ is separable, $X$ is smooth over $k$. Let $U:=X \cap\{P(t) \neq 0\}$. By the local description of its universal torsors [17, Proposition 2] (see also [17, proof of Proposition 3]), we have

$$
\mathcal{T}_{U} \subset \mathbb{A}_{k}^{1} \times R_{K / k}\left(\mathbb{G}_{\mathrm{m}, K}\right)^{2}
$$

defined by

$$
t-\sqrt{a}=\rho \cdot N_{K / L}(\mathbf{x}) \cdot \sigma\left(N_{K / L}(\mathbf{y})\right) \neq 0
$$

for $k \subset L:=k(\sqrt{a}) \subset K$, some $(\rho, \xi) \in L^{\times} \times K^{\times}$satisfying

$$
c N_{L / k}(\rho)=N_{K / k}(\xi),
$$

and $\sigma \in \Gamma_{k}$ with $\sigma(\sqrt{a})=-\sqrt{a}$. The restriction of $f: \mathcal{T} \rightarrow X$ to $\mathcal{T}_{U}$ is given by

$$
(t, \mathbf{x}, \mathbf{y}) \mapsto(t, \xi \mathbf{x y})
$$

We write

$$
K=L(\sqrt{u+v \sqrt{a}})
$$

with $u, v \in k$. For $\mathbf{x}=\left(x_{1}+x_{2} \sqrt{a}\right)+\left(x_{3}+x_{4} \sqrt{a}\right) \sqrt{u+v \sqrt{a}} \in K$ and $\mathbf{y} \in K$, we have

$$
\begin{aligned}
N_{K / L}(\mathbf{x}) & =\left(x_{1}+x_{2} \sqrt{a}\right)^{2}-\left(x_{3}+x_{4} \sqrt{a}\right)^{2}(u+v \sqrt{a}) \\
& =g_{0}(\mathbf{x})+g_{1}(\mathbf{x}) \sqrt{a} \\
\rho \cdot \sigma\left(N_{K / L}(\mathbf{y})\right) & =\lambda_{0}(\mathbf{y})+\lambda_{1}(\mathbf{y}) \sqrt{a}
\end{aligned}
$$

for quadratic forms

$$
\begin{aligned}
& g_{0}(\mathbf{x})=x_{1}^{2}+a x_{2}^{2}-u x_{3}^{2}-a u x_{4}^{2}-2 a v x_{3} x_{4}, \\
& g_{1}(\mathbf{x})=2 x_{1} x_{2}-2 u x_{3} x_{4}-v x_{3}^{2}-a v x_{4}^{2}
\end{aligned}
$$

in $\mathbf{x}=\left(x_{1}, \ldots, x_{4}\right)$ and some quadratic forms $\lambda_{0}(\mathbf{y}), \lambda_{1}(\mathbf{y})$ in $\mathbf{y}=\left(y_{1}, \ldots, y_{4}\right)$. Then

$$
\rho \cdot N_{K / L}(\mathbf{x}) \cdot \sigma\left(N_{K / L}(\mathbf{y})\right)=g_{0}(\mathbf{x}) \lambda_{0}(\mathbf{y})+a g_{1}(\mathbf{x}) \lambda_{1}(\mathbf{y})+\left(g_{0}(\mathbf{x}) \lambda_{1}(\mathbf{y})+g_{1}(\mathbf{x}) \lambda_{0}(\mathbf{y})\right) \sqrt{a} .
$$

This gives an open embedding of $\mathcal{T}_{U}$ into the affine variety $\widetilde{Y} \subset \mathbb{A}_{k}^{9}$ with coordinates $(t, \mathbf{x}, \mathbf{y})$ defined by

$$
t=g_{0}(\mathbf{x}) \lambda_{0}(\mathbf{y})+a g_{1}(\mathbf{x}) \lambda_{1}(\mathbf{y}), \quad-1=g_{0}(\mathbf{x}) \lambda_{1}(\mathbf{y})+g_{1}(\mathbf{x}) \lambda_{0}(\mathbf{y}) .
$$

Clearly, $\widetilde{Y} \cong Y$ for $Y \subset \mathbb{A}_{k}^{8}$ with coordinates (x, y) defined by

$$
-1=g_{0}(\mathbf{x}) \lambda_{1}(\mathbf{y})+g_{1}(\mathbf{x}) \lambda_{0}(\mathbf{y}) .
$$

We have a morphism $g: Y \rightarrow X$ defined by

$$
(\mathbf{x}, \mathbf{y}) \mapsto\left(g_{0}(\mathbf{x}) \lambda_{0}(\mathbf{y})+a g_{1}(\mathbf{x}) \lambda_{1}(\mathbf{y}), \xi \mathbf{x y}\right) .
$$

We observe that $g: Y \rightarrow X$ and $f: \mathcal{T} \rightarrow X$ have the same restriction to $\mathcal{T}_{U}$. 
Note that $X$ is smooth over $k$. Also $Y$ is smooth over $k$ since

$$
\bar{Y} \cong\left\{2 \sqrt{a}=\sigma(\rho) w_{1} w_{2} w_{3} w_{4}-\rho w_{1}^{\prime} w_{2}^{\prime} w_{3}^{\prime} w_{4}^{\prime}\right\} \subset \mathbb{A} \frac{8}{k},
$$

using (4.1). By [17, Proposition 2], $\operatorname{Pic}(\bar{X})$ is torsion-free of finite rank, hence $\mathcal{T}_{U}$ is a torsor under a torus over the geometrically integral variety $U$, and hence $\mathcal{T}_{U}$ is geometrically integral.

Therefore, we can apply Lemma 3.1 to get strong approximation with Brauer-Manin obstruction for $X$ off $v_{0}$ once we have shown that $Y$ satisfies strong approximation off $v_{0}$, which is done in Proposition 4.1 below.

The following result completes the proof of Theorem 1.1.

Proposition 4.1. The affine variety $Y \subset \mathbb{A}_{k}^{8}$ defined by (4.2) satisfies strong approximation off $v_{0}$.

Proof. Consider the projection $\pi: Y \rightarrow \mathbb{A}_{k}^{4}$ to the $\mathbf{y}$-coordinate. In (4.2), we can write

$$
g_{0}(\mathbf{x}) \lambda_{1}(\mathbf{y})+g_{1}(\mathbf{x}) \lambda_{0}(\mathbf{y})=q_{0}\left(x_{1}, x_{2}\right)+q_{1}\left(x_{3}, x_{4}\right),
$$

where $q_{0}, q_{1}$ are the following binary quadratic forms with coefficients in $k\left[y_{1}, \ldots, y_{4}\right]$ :

$$
\begin{gathered}
q_{0}\left(x_{1}, x_{2}\right)=\lambda_{1}(\mathbf{y}) x_{1}^{2}+2 \lambda_{0}(\mathbf{y}) x_{1} x_{2}+a \lambda_{1}(\mathbf{y}) x_{2}^{2}, \\
q_{1}\left(x_{3}, x_{4}\right)=-\left(u \lambda_{1}(\mathbf{y})+v \lambda_{0}(\mathbf{y})\right) x_{3}^{2}-2\left(a v \lambda_{1}(\mathbf{y})+u \lambda_{0}(\mathbf{y})\right) x_{3} x_{4} \\
-a\left(u \lambda_{1}(\mathbf{y})+v \lambda_{0}(\mathbf{y})\right) x_{4}^{2} .
\end{gathered}
$$

Its discriminants are

$$
\begin{aligned}
\operatorname{disc}\left(q_{0}\right) & =a \lambda_{1}(\mathbf{y})^{2}-\lambda_{0}(\mathbf{y})^{2} \\
& =-N_{L / k}(\rho) N_{K / k}(\mathbf{y}) \\
\operatorname{disc}\left(q_{1}\right) & =\left(u^{2}-a v^{2}\right)\left(a \lambda_{1}(\mathbf{y})^{2}-\lambda_{0}(\mathbf{y})^{2}\right) \\
& =-N_{L / k}(u+v \sqrt{a}) N_{L / k}(\rho) N_{K / k}(\mathbf{y}) .
\end{aligned}
$$

For $N_{K / k}(\mathbf{y}) \neq 0$, the binary forms $q_{0}, q_{1}$ have full rank, hence each fiber $Y_{\mathbf{y}}$ is a three-dimensional quadric.

Denote

$$
Z:=\left\{\lambda_{1}(\mathbf{y})=\operatorname{disc}\left(q_{0}\right)=0\right\} \cup\left\{u \lambda_{1}(\mathbf{y})+v \lambda_{0}(\mathbf{y})=\operatorname{disc}\left(q_{1}\right)=0\right\},
$$

which is a closed subset of $\mathbb{A}_{k}^{4}$. For strong approximation, by smoothness of $Y$, it is enough to show that its open subset $\pi^{-1}\left(\mathbb{A}_{k}^{4} \backslash Z\right)$ satisfies strong approximation off $v_{0}$. Applying the natural fibration $\pi^{-1}\left(\mathbb{A}_{k}^{4} \backslash Z\right)$ over $\mathbb{A}_{k}^{4} \backslash Z$, we only need to check the conditions for an application of the version of the fibration method stated in [15, Proposition 3.1].

It is clear that all geometric fibers of $\pi^{-1}\left(\mathbb{A}_{k}^{4} \backslash Z\right)$ over $\mathbb{A}_{k}^{4} \backslash Z$ are non-empty and integral. Recall that $\operatorname{disc}\left(q_{0}\right)$ and $\operatorname{disc}\left(q_{1}\right)$ differ only by a constant in $k^{\times}$. Since $\operatorname{disc}\left(q_{0}\right)$ is irreducible of degree 4 and $\lambda_{1}(\mathbf{y})$ has degree 2, and similarly for the second part, we know that $Z$ has dimension 2. Therefore $\mathbb{A}_{k}^{4} \backslash Z$ satisfies strong approximation off $v_{0}$ by [31, Lemma 1.1], hence the condition (i) holds.

Let $W:=\left\{N_{K / k}(\mathbf{y}) \neq 0\right\} \subset \mathbb{A}_{k}^{4} \backslash Z$. In the following, we will check the conditions (ii) and (iii). 
For $v_{0}$ as above, we claim that $Y_{\mathbf{y}}\left(k_{v_{0}}\right)$ is not compact for any $\mathbf{y} \in W\left(k_{v_{0}}\right)$, hence the condition (iii) holds. For any $k$-point $r$ in $W$, the fiber $Y_{r}$ is a quadric of dimension 3. Since $Y_{r}\left(k_{v_{0}}\right)$ is not compact, $Y_{r}$ satisfies strong approximation off $v_{0}$ (see for example [14, Theorem 3.7, Section 5.3]), hence the condition (ii) holds.

In the following we prove the claim. If $v_{0}$ is complex, this claim is obvious, so we assume that $v_{0}$ is real and consider everything in the following with respect to the corresponding real embedding of $k$. If one of $\operatorname{disc}\left(q_{0}\right)$ and $\operatorname{disc}\left(q_{1}\right)$ is negative, then one of $q_{0}$ and $q_{1}$ is indefinite, and the claim is true. So we can assume $\operatorname{disc}\left(q_{0}\right), \operatorname{disc}\left(q_{1}\right)>0$, and we claim that one of $q_{0}, q_{1}$ is positive definite and the other is negative definite, i.e., $\lambda_{1}(\mathbf{y})\left(-\left(u \lambda_{1}(\mathbf{y})+v \lambda_{0}(\mathbf{y})\right)\right)<0$.

For this, we show first that $u>0$. By assumption, $a \lambda_{1}(\mathbf{y})^{2}-\lambda_{0}(\mathbf{y})^{2}>0$ (so $a>0$ ) and $u^{2}-a v^{2}>0$ (so $u-v \sqrt{a}$ and $u+v \sqrt{a}$ have the same sign). If $u<0$, then both $u-v \sqrt{a}$ and $u+v \sqrt{a}$ are negative, so all places of $K$ above $v_{0}$ are complex, and $N_{K / k}$ is positive definite. By unboundedness of $p\left(X\left(k_{v_{0}}\right)\right)$, we have $c>0$. Therefore, $N_{L / k}(\rho)=c^{-1} N_{K / k}(\xi)>0$, hence $\operatorname{disc}\left(q_{0}\right)<0$, contradicting our assumption of definiteness of $q_{0}$. Hence $u>0$.

Therefore, $u^{2}-a v^{2}>0$ implies $u>|v| \sqrt{a}$. Furthermore, $a \lambda_{1}(\mathbf{y})^{2}-\lambda_{0}(\mathbf{y})^{2}>0$ implies $\left|\lambda_{1}(\mathbf{y})\right|>\left|\lambda_{0}(\mathbf{y})\right| / \sqrt{a}$. Therefore,

$$
\begin{aligned}
\lambda_{1}(\mathbf{y})\left(u \lambda_{1}(\mathbf{y})+v \lambda_{0}(\mathbf{y})\right) & >(\sqrt{a}|v|) \frac{\left|\lambda_{0}(\mathbf{y})\right|}{\sqrt{a}}\left|\lambda_{1}(\mathbf{y})\right|+v \lambda_{0}(\mathbf{y}) \lambda_{1}(\mathbf{y}) \\
& =\left|v \lambda_{0}(\mathbf{y}) \lambda_{1}(\mathbf{y})\right|+v \lambda_{0}(\mathbf{y}) \lambda_{1}(\mathbf{y}) \geq 0,
\end{aligned}
$$

so one of $q_{0}, q_{1}$ is positive definite and the other is negative definite, and $Y_{\mathbf{y}}\left(k_{v_{0}}\right)$ is not compact also in this case.

Our next goal is to compute the Brauer group of $X$ as in Theorem 1.1. We start with some lemmas that will also be used in our counterexample in Section 6.

Let $X \subset \mathbb{A}_{k}^{n+1}$ be defined by (1.1) for $s=1$, a finite extension $K / k$ of fields of characteristic 0 and a non-constant polynomial $P(t) \in k[t]$. Recall that $X^{\mathrm{sm}} \subset X$ is the smooth locus of $X$. If $P(t)$ is separable, then $X^{\text {sm }}=X$.

The following lemma shows that $\operatorname{Br}\left(\bar{X}^{\mathrm{sm}}\right)=0$.

Lemma 4.2. Let $k$ be an algebraically closed field of characteristic 0 . Let

$$
P(t)=c\left(t-a_{1}\right)^{e_{1}} \cdots\left(t-a_{r}\right)^{e_{r}} \in k[t]
$$

with $r \geq 1, \operatorname{gcd}\left(e_{1}, \ldots, e_{r}\right)=1$ and $a_{i} \neq a_{j}$ for $i \neq j$. Let $X \subset \mathbb{A}^{n+1}$ be the affine variety defined by $P(t)=z_{1} \cdots z_{n}$. Then $\operatorname{Br}\left(X^{\mathrm{sm}}\right)=0$.

Proof. We prove the claim by induction on $n$. If $n=1$, then $X^{\mathrm{sm}}=X \cong \mathbb{A}_{k}^{1}$. By Tsen's theorem, $\operatorname{Br}\left(X^{\mathrm{sm}}\right)=0$.

For $n>1$, consider the projection

$$
X \rightarrow \mathbb{A}_{k}^{1}, \quad\left(t, z_{1}, \ldots, z_{n}\right) \mapsto z_{n} .
$$

Let $\eta$ be the generic point of $\mathbb{A}_{k}^{1}$. Then the generic fiber $X_{\eta}^{\mathrm{sm}}$ is just the smooth locus of the affine variety over $k\left(z_{n}\right)$ defined by

$$
\frac{1}{z_{n}} P(t)=z_{1} \cdots z_{n-1}
$$

We have $\operatorname{Br}\left(X^{\mathrm{sm}}\right) \subset \operatorname{Br}\left(X_{\eta}^{\mathrm{sm}}\right)$ for any smooth variety, and we will show that the latter is trivial. 
Let $X_{\bar{\eta}}^{\mathrm{sm}}=X_{\eta}^{\mathrm{sm}} \times{ }_{k\left(z_{n}\right)} \overline{k\left(z_{n}\right)}$. Since $k\left[X_{\bar{\eta}}^{\mathrm{sm}}\right]^{\times}=\overline{k\left(z_{n}\right)} \times$ by the same residue computation as in the proof of [17, Proposition 2], we have $H^{2}\left(k\left(z_{n}\right), k\left[X_{\bar{\eta}}\right]^{\times}\right)=\operatorname{Br}\left(k\left(z_{n}\right)\right)$. Therefore, the Hochschild-Serre spectral sequence gives the exact sequence

$$
\operatorname{Br}\left(k\left(z_{n}\right)\right) \rightarrow \operatorname{ker}\left(\operatorname{Br}\left(X_{\eta}^{\mathrm{sm}}\right) \rightarrow \operatorname{Br}\left(X_{\bar{\eta}}^{\mathrm{sm}}\right)\right) \rightarrow H^{1}\left(k\left(z_{n}\right), \operatorname{Pic}\left(X_{\bar{\eta}}^{\mathrm{sm}}\right)\right) .
$$

By Tsen's theorem, $\operatorname{Br}\left(k\left(z_{n}\right)\right)=0$ since $k=\bar{k}$. By the induction hypothesis, $\operatorname{Br}\left(X_{\bar{\eta}}^{\mathrm{sm}}\right)=0$.

Since $\operatorname{gcd}\left(e_{1}, \ldots, e_{r}\right)=1$, the Picard group $\operatorname{Pic}\left(X_{\bar{\eta}}^{\mathrm{sm}}\right)$ is a constant torsion-free module (i.e., the natural action of $\Gamma_{k\left(z_{n}\right)}$ on it is trivial), hence we have $H^{1}\left(k\left(z_{n}\right), \operatorname{Pic}\left(X_{\bar{\eta}}^{\mathrm{sm}}\right)\right)=0$. Therefore, the exact sequence above gives $\operatorname{Br}\left(X_{\eta}^{\mathrm{sm}}\right)=0$.

For $X$ as above, let $U \subset X^{\mathrm{sm}}$ be the open subset defined by $P(t) \neq 0$. Denote

$$
\widehat{T}=\bar{k}[U]^{\times} / \bar{k}^{\times} \quad \text { and } \quad \widehat{M}=\operatorname{Div}_{\bar{X} \backslash \bar{U}}(\bar{X}) .
$$

We have the natural exact sequence

$$
0 \rightarrow \widehat{T} \rightarrow \widehat{M} \rightarrow \operatorname{Pic}\left(\bar{X}^{\mathrm{sm}}\right) \rightarrow 0 .
$$

Lemma 4.3. Let $k$ be a field of characteristic 0 with $H^{3}\left(k, \bar{k}^{\times}\right)=0$. Assume that

$$
P(t)=c g_{1}(t)^{e_{1}} \cdots g_{r}(t)^{e_{r}}
$$

for $c \in k^{\times}$, pairwise distinct irreducible monic polynomials $g_{1}(t), \ldots, g_{r}(t) \in k[t]$ and positive integers $e_{1}, \ldots, e_{r}$ with $\operatorname{gcd}\left(e_{1}, \ldots, e_{r}\right)=1$. Let $X$ be defined by $(1.1)$ with $s=1$. Then

$$
\operatorname{Br}\left(X^{\mathrm{sm}}\right) / \operatorname{Br}_{0}\left(X^{\mathrm{sm}}\right) \cong H^{1}\left(k, \operatorname{Pic}\left(\bar{X}^{\mathrm{sm}}\right)\right) \cong \operatorname{ker}\left(H^{2}(k, \widehat{T}) \rightarrow H^{2}(k, \widehat{M})\right) .
$$

Proof. By Lemma 4.2, we have $\operatorname{Br}\left(\bar{X}^{\mathrm{sm}}\right)=0$, hence

$$
\operatorname{Br}\left(X^{\mathrm{sm}}\right)=\operatorname{Br}_{1}\left(X^{\mathrm{sm}}\right) \text {. }
$$

Note that $\bar{k}\left[X^{\mathrm{sm}}\right]^{\times}=\bar{k}^{\times}$.

By the Hochschild-Serre spectral sequence, we have the exact sequence

$$
\operatorname{Br}(k) \rightarrow \operatorname{Br}_{1}\left(X^{\mathrm{sm}}\right) \rightarrow H^{1}\left(k, \operatorname{Pic}\left(\bar{X}^{\mathrm{sm}}\right)\right) \rightarrow H^{3}\left(k, \bar{k}^{\times}\right)=0,
$$

hence $\operatorname{Br}\left(X^{\mathrm{sm}}\right) / \operatorname{Br}_{0}\left(X^{\mathrm{sm}}\right) \cong H^{1}\left(k, \operatorname{Pic}\left(\bar{X}^{\mathrm{sm}}\right)\right)$.

Since $\widehat{M}$ is a permutation $\Gamma_{k}$-module, $H^{1}(k, \widehat{M})=0$. Hence (4.3) implies that

$$
0 \rightarrow H^{1}\left(k, \operatorname{Pic}\left(\bar{X}^{\mathrm{sm}}\right)\right) \rightarrow H^{2}(k, \widehat{T}) \rightarrow H^{2}(k, \hat{M})
$$

is exact, and the result follows.

To compute the Brauer group in the situation of Theorem 1.1, we argue similarly as in Lemma 4.3. The condition $H^{3}\left(k, \bar{k}^{\times}\right)=0$ holds when $k$ is a number field, for example.

Proposition 4.4. Let $k$ be a field of characteristic 0 with $H^{3}\left(k, \bar{k}^{\times}\right)=0$. Let $K / k$ be of degree 4 and $P(t)=c\left(t^{2}-a\right)$ irreducible over $k$ and split over $K$. Let $X$ be defined by (1.1) with $s=1$. We have

$$
\operatorname{Br}(X) / \operatorname{Br}_{0}(X)= \begin{cases}0, & \text { if } K / k \text { cyclic or non-Galois, } \\ \mathbb{Z} / 2 \mathbb{Z}, & \text { otherwise }\end{cases}
$$


Proof. First, suppose that $K / k$ is Galois. Since $\operatorname{Pic}(\bar{X})$ is split by $K$ and torsion-free, we have

$$
H^{1}(k, \operatorname{Pic}(\bar{X})) \cong H^{1}\left(K / k, \operatorname{Pic}\left(X_{K}\right)\right)
$$

by the inflation-restriction sequence.

Let $L:=k(\sqrt{a})$. Note that $\operatorname{Div}_{X_{K} \backslash U_{K}}\left(X_{K}\right) \cong \mathbb{Z}[L / k] \otimes \mathbb{Z}[K / k]$, hence

$$
H^{i}\left(K / k, \operatorname{Div}_{X_{K} \backslash U_{K}}\left(X_{K}\right)\right)=0
$$

for $i>0$. With the exact sequence

$$
0 \rightarrow K[U]^{\times} / K^{\times} \rightarrow \operatorname{Div}_{X_{K} \backslash U_{K}}\left(X_{K}\right) \rightarrow \operatorname{Pic}\left(X_{K}\right) \rightarrow 0,
$$

this gives $H^{1}\left(K / k, \operatorname{Pic}\left(X_{K}\right)\right) \cong H^{2}\left(K / k, K[U]^{\times} / K^{\times}\right)$.

To compute the latter, we consider the exact sequence

$$
0 \rightarrow \mathbb{Z} \rightarrow \mathbb{Z}[L / k] \oplus \mathbb{Z}[K / k] \rightarrow K[U]^{\times} / K^{\times} \rightarrow 0
$$

from [17, Proposition 2]. We consider

$$
\begin{aligned}
H^{2}(K / k, \mathbb{Z}) & \rightarrow H^{2}(K / k, \mathbb{Z}[L / k] \oplus \mathbb{Z}[K / k]) \rightarrow H^{2}\left(K / k, K[U]^{\times} / K^{\times}\right) \\
& \rightarrow H^{3}(K / k, \mathbb{Z}) \rightarrow H^{3}(K / k, \mathbb{Z}[L / k] \oplus \mathbb{Z}[K / k]) .
\end{aligned}
$$

We have $H^{2}(K / k, \mathbb{Z}[K / k])=0$ since this is an induced module, and

$$
H^{2}(K / k, \mathbb{Z}[L / k]) \cong H^{2}(K / L, \mathbb{Z})
$$

by Shapiro's lemma. Hence the first map is the natural map $H^{2}(K / k, \mathbb{Z}) \rightarrow H^{2}(K / L, \mathbb{Z})$, which is surjective since any quartic Galois extension $K / k$ is abelian.

Similarly, we have $H^{3}(K / k, \mathbb{Z}[K / k])=0$ and $H^{3}(K / k, \mathbb{Z}[L / k]) \cong H^{3}(K / L, \mathbb{Z})$. Since $\operatorname{Gal}(K / L)$ is cyclic,

$$
H^{3}(K / L, \mathbb{Z}) \cong H^{1}(K / L, \mathbb{Z}) \cong \operatorname{Hom}(\operatorname{Gal}(K / L), \mathbb{Z})=0 .
$$

Therefore, $H^{3}(K / k, \mathbb{Z}[L / k] \oplus \mathbb{Z}[K / k])=0$.

Therefore, the third map in our part of the long exact sequence above is an isomorphism. Now

$$
H^{3}(K / k, \mathbb{Z})= \begin{cases}0, & \text { if } K / k \text { is cyclic, } \\ \mathbb{Z} / 2 \mathbb{Z}, & \text { if } \operatorname{Gal}(K / k) \cong \mathbb{Z} / 2 \mathbb{Z} \times \mathbb{Z} / 2 \mathbb{Z} .\end{cases}
$$

Indeed, in the first case, we argue as in the previous paragraph. In the second case, we can refer to a classical computation of Schur (see [24, Corollary 2.2.12]).

Next, suppose that $K / k$ is non-Galois. By the inflation-restriction sequence, we have

$$
0 \rightarrow H^{1}\left(L / k, \operatorname{Pic}(\bar{X})^{\Gamma_{L}}\right) \rightarrow H^{1}(k, \operatorname{Pic}(\bar{X})) \rightarrow H^{1}(L, \operatorname{Pic}(\bar{X})) .
$$

We will show that the first and third groups are trivial.

For the triviality of $H^{1}\left(L / k, \operatorname{Pic}(\bar{X})^{\Gamma_{L}}\right)$, we note that (4.3) gives

$$
0 \rightarrow \widehat{T}^{\Gamma_{L}} \rightarrow \widehat{M}^{\Gamma_{L}} \rightarrow \operatorname{Pic}(\bar{X})^{\Gamma_{L}} \rightarrow H^{1}\left(\Gamma_{L}, \widehat{T}\right) .
$$

Analogously to (4.5), we have over $\bar{k}$ the exact sequence

$$
0 \rightarrow \mathbb{Z} \rightarrow \mathbb{Z}[L / k] \oplus \mathbb{Z}[K / k] \rightarrow \widehat{T} \rightarrow 0 .
$$

Since the action of $\Gamma_{L}$ on $\mathbb{Z}[L / k]$ is trivial, this exact sequence has a $\Gamma_{L}$-equivariant splitting, hence $\widehat{T}$ is a permutation $\Gamma_{L}$-module, so $H^{1}(L, \widehat{T})=0$. 
Therefore, (4.7) is a short exact sequence, giving

$$
H^{1}\left(L / k, \hat{M}^{\Gamma_{L}}\right) \rightarrow H^{1}\left(L / k, \operatorname{Pic}(\bar{X})^{\Gamma_{L}}\right) \rightarrow H^{2}\left(L / k, \widehat{T}^{\Gamma_{L}}\right) \rightarrow H^{2}\left(L / k, \hat{M}^{\Gamma_{L}}\right) .
$$

We note that $\hat{M} \cong \mathbb{Z}[L / k] \otimes \mathbb{Z}[K / k]$, hence $\hat{M}^{\Gamma_{L}} \cong \mathbb{Z}[L / k] \otimes \mathbb{Z}[K / k]^{\Gamma_{L}}$ is an induced $\operatorname{Gal}(L / k)$-module. Therefore, $H^{i}\left(L / k, \hat{M}^{\Gamma_{L}}\right)=0$ for $i>0$, hence the second map in our part of the long exact sequence is an isomorphism.

We have

$$
\mathbb{Z}[K / k]^{\Gamma_{L}} \cong \mathbb{Z}[L / k] .
$$

We choose $\sigma \in \Gamma_{k}$ and $\beta \in \Gamma_{L}$ such that $\Gamma_{k} / \Gamma_{L}=\left\{\Gamma_{L}, \sigma \Gamma_{L}\right\}$ and $\Gamma_{L} / \Gamma_{K}=\left\{\Gamma_{K}, \beta \Gamma_{K}\right\}$. Then

$$
\Gamma_{k} / \Gamma_{K}=\left\{\Gamma_{K}, \sigma \Gamma_{K}, \beta \Gamma_{K}, \sigma \beta \Gamma_{K}\right\} .
$$

Now the orbits of the natural action of $\Gamma_{L}=\Gamma_{K} \cup \beta \Gamma_{K}$ on this are $\left\{\Gamma_{K}, \beta \Gamma_{K}\right\}$ (since $\Gamma_{K}$ is normal in $\Gamma_{L}$ because of $[K: L]=2$ ) and $\left\{\sigma \Gamma_{K}, \sigma \beta \Gamma_{K}\right\}$ (since the action of $\Gamma_{L}$ on this set is non-trivial because $\sigma \beta \sigma^{-1}$ maps $\sigma \Gamma_{K}$ to $\sigma \beta \Gamma_{K}$ and lies in $\Gamma_{L}$ because $\Gamma_{L}$ is normal in $\Gamma_{k}$ since $\left.[L: k]=2\right)$. Therefore, $\mathbb{Z}[K / k]^{\Gamma_{L}}=\left\langle\Gamma_{K}+\beta \Gamma_{K}, \sigma \Gamma_{K}+\sigma \beta \Gamma_{K}\right\rangle$ is of rank 2, with a non-trivial action of $\operatorname{Gal}(L / k)$.

Taking $\Gamma_{L}$-invariants of (4.8) gives

$$
0 \rightarrow \mathbb{Z} \rightarrow \mathbb{Z}[L / k] \oplus \mathbb{Z}[L / k] \rightarrow \hat{T}^{\Gamma_{L}} \rightarrow H^{1}(L, \mathbb{Z})=0 .
$$

The long exact sequence gives

$$
0=H^{2}(L / k, \mathbb{Z}[L / k] \oplus \mathbb{Z}[L / k]) \rightarrow H^{2}\left(L / k, \widehat{T}^{\Gamma_{L}}\right) \rightarrow H^{3}(L / k, \mathbb{Z})=0,
$$

where the latter is trivial because $L / k$ is cyclic. Hence $H^{2}\left(L / k, \widehat{T}^{\Gamma_{L}}\right)=0$, which implies $H^{1}\left(L / k, \operatorname{Pic}(\bar{X})^{\Gamma_{L}}\right)=0$ in (4.6).

For the triviality of $H^{1}(L, \operatorname{Pic}(\bar{X}))$ in (4.6), we write

$$
K=k(\sqrt{u+v \sqrt{a}})
$$

for some $u \in k$ and $v \in k^{\times}$. Then $K \otimes_{k} L \cong K \oplus K^{\prime}$ with $K^{\prime}=k(\sqrt{u-v \sqrt{a}})$. We note that $X \times_{k} L$ is defined by the equation

$$
c(t-\sqrt{a})(t+\sqrt{a})=N_{K / L}\left(\mathbf{z}_{1}\right) N_{K^{\prime} / L}\left(\mathbf{z}_{2}\right) .
$$

Over $\bar{k}$, we have $N_{K / L}\left(\mathbf{z}_{1}\right)=u_{1} u_{2}$ and $N_{K^{\prime} / L}\left(\mathbf{z}_{2}\right)=u_{3} u_{4}$.

As a sequence of $\Gamma_{L}$-modules, (4.8) becomes

$$
0 \rightarrow \mathbb{Z} \rightarrow \mathbb{Z}^{2} \oplus \mathbb{Z}[K / L] \oplus \mathbb{Z}\left[K^{\prime} / L\right] \rightarrow \widehat{T} \rightarrow 0,
$$

hence

$$
\widehat{T} \cong \mathbb{Z} \oplus \mathbb{Z}[K / L] \oplus \mathbb{Z}\left[K^{\prime} / L\right],
$$

with basis $\left(t-\sqrt{a}, u_{1}, u_{2}, u_{3}, u_{4}\right)$. Therefore,

$$
H^{2}(L, \widehat{T}) \cong H^{2}(L, \mathbb{Z}) \oplus H^{2}(K, \mathbb{Z}) \oplus H^{2}\left(K^{\prime}, \mathbb{Z}\right)
$$

by Shapiro's lemma.

Then

$$
D_{i}^{+}=\left\{t=\sqrt{a}, u_{i}=0\right\}, \quad D_{i}^{-}=\left\{t=-\sqrt{a}, u_{i}=0\right\}
$$


for $i=1, \ldots, 4$ are a basis of $\hat{M}$. Considering the action of $\Gamma_{L}$ on this basis shows that

$$
\begin{aligned}
& \mathbb{Z} D_{1}^{+} \oplus \mathbb{Z} D_{2}^{+} \cong \mathbb{Z} D_{1}^{-} \oplus \mathbb{Z} D_{2}^{-} \cong \mathbb{Z}[K / L], \\
& \mathbb{Z} D_{3}^{+} \oplus \mathbb{Z} D_{4}^{+} \cong \mathbb{Z} D_{3}^{-} \oplus \mathbb{Z} D_{4}^{-} \cong \mathbb{Z}\left[K^{\prime} / L\right],
\end{aligned}
$$

hence $\hat{M} \cong\left(\mathbb{Z}[K / L] \oplus \mathbb{Z}\left[K^{\prime} / L\right]\right)^{2}$. Therefore,

$$
H^{2}(L, \widehat{M}) \cong\left(H^{2}(K, \mathbb{Z}) \oplus H^{2}\left(K^{\prime}, \mathbb{Z}\right)\right)^{2},
$$

again by Shapiro's lemma.

The map

$$
\widehat{T} \rightarrow \widehat{M}, \quad t-\sqrt{a} \mapsto D_{1}^{+}+D_{2}^{+}+D_{3}^{+}+D_{4}^{+}, u_{i} \mapsto D_{i}^{+}+D_{i}^{-}
$$

from (4.3) induces a map $H^{2}(L, \widehat{T}) \rightarrow H^{2}(L, \widehat{M})$ that can be explicitly described as

$$
\left(\chi_{1}, \chi_{2}, \chi_{3}\right) \mapsto\left(\chi_{1 \mid K}+\chi_{2}, \chi_{1 \mid K^{\prime}}+\chi_{3}, \chi_{2}, \chi_{3}\right)
$$

The sequence (4.3) gives

$$
0=H^{1}(L, \widehat{M}) \rightarrow H^{1}(L, \operatorname{Pic}(\bar{X})) \rightarrow H^{2}(L, \widehat{T}) \stackrel{\phi}{\rightarrow} H^{2}(L, \widehat{M})
$$

since $\widehat{M}$ is a permutation $\Gamma_{L^{-}}$-module. To deduce triviality of $H^{1}(L, \operatorname{Pic}(\bar{X}))$, we show that $\phi$ is injective. If $\left(\chi_{1}, \chi_{2}, \chi_{3}\right) \in \operatorname{ker} \phi$, then $\chi_{2}, \chi_{3}$ and hence also $\chi_{1 \mid K}, \chi_{1 \mid K^{\prime}}$ are trivial. Since $K \cap K^{\prime}=L$, the latter implies $\chi_{1}=0$, proving injectivity of $\phi$.

Using Lemma 4.3 and (4.6), we deduce

$$
\operatorname{Br}(X) / \operatorname{Br}_{0}(X) \cong H^{1}(k, \operatorname{Pic}(\bar{X}))=0 .
$$

Corollary 4.5. In the situation of Theorem 1.1, assume additionally that the extension $K / k$ is cyclic or non-Galois. Then strong approximation holds for $X$ off $v_{0}$.

Proof. This follows directly from Theorem 1.1 and Proposition 4.4.

Corollary 4.6. Let $K / k$ be an extension of number fields of degree 4 , and let

$$
\omega_{1}, \ldots, \omega_{4} \in \mathcal{O}_{K}
$$

be a basis of $K$ over $k$. Let $\mathfrak{X} \subset \mathbb{A}_{\mathcal{O}_{k}}^{5}$ be the affine scheme defined by

$$
a t^{2}-b=N_{K / k}\left(z_{1} \omega_{1}+\cdots+z_{4} \omega_{4}\right)
$$

for some $a, b \in \mathcal{O}_{k}$ such that $a t^{2}-b \in k[t]$ is irreducible and splits in $K$. For $X:=\mathfrak{X} \times{ }_{\mathcal{O}_{k}} k$ and $p: X \rightarrow \mathbb{A}_{k}^{1}$ the projection to the $t$-coordinate, assume that $\prod_{v \in \infty_{k}} p\left(X\left(k_{v}\right)\right)$ is not bounded. If

$$
\left(\prod_{v \in \infty_{k}} X\left(k_{v}\right) \times \prod_{v \notin \infty_{k}} \mathfrak{X}\left(\mathcal{O}_{v}\right)\right)^{\operatorname{Br}(X)} \neq \emptyset,
$$

then $\mathfrak{X}\left(\mathcal{O}_{k}\right) \neq \emptyset$, i.e., the Hasse principle with Brauer-Manin obstruction holds for integral points on $\mathfrak{X}$. In particular, if $K / k$ is cyclic or non-Galois, then the Hasse principle holds for integral points on $\mathfrak{X}$.

Proof. This follows from Theorem 1.1 as in Remark 2.2. For $K / k$ cyclic or non-Galois, we have $\operatorname{Br}(X)=\operatorname{Br}_{0}(X)$ by Proposition 4.4. 


\section{Totally split polynomials represented by norms}

For the proof of strong approximation for $X$ as in Theorem 1.2, we determine its universal torsors in Proposition 5.1 below. If the factors of $P(\mathbf{t})$ are linear forms, these turn out to be essentially the varieties $\mathscr{V}$ that satisfy weak approximation by [4, Theorem 1.3] based on [4, Theorem 5.2]; for Theorem 1.2, we use a generalization, Theorem 5.2 below. By our Descent Lemma 3.1, it then remains to prove strong approximation for $\mathscr{V}$, which we do in Proposition 5.3.

Proposition 5.1. Let $k$ be a field of characteristic 0 . Let $K / k$ be a field extension of degree $n$. Let $P(\mathbf{t})=c g_{1}(\mathbf{t})^{e_{1}} \cdots g_{r}(\mathbf{t})^{e_{r}}$ with $c \in k^{\times}$and pairwise non-proportional linear polynomials $g_{i}(\mathbf{t}) \in k\left[t_{1}, \ldots, t_{s}\right]$ in $s \geq 2$ variables, and $e_{1}, \ldots, e_{r} \in \mathbb{Z}_{>0}$. Let $X$ be defined by (1.1). Universal torsors over $X^{\mathrm{sm}}$ exist if and only if there are $\lambda_{1}, \ldots, \lambda_{r} \in k^{\times}$and $\xi \in K^{\times}$ satisfying

$$
c \lambda_{1}^{e_{1}} \cdots \lambda_{r}^{e_{r}}=N_{K / k}(\xi) .
$$

Then restrictions $\mathcal{T}_{U} \subset \mathbb{A}_{k}^{r n+s}$ of universal torsors $f: \mathcal{T} \rightarrow X^{\mathrm{sm}}$ to $U:=X \cap\{P(\mathbf{t}) \neq 0\}$ are geometrically rational and defined by

$$
N_{K / k}\left(\mathbf{z}_{i}\right)=\lambda_{i}^{-1} g_{i}(\mathbf{t}) \neq 0,
$$

for $i=1, \ldots, r$, for some $\lambda_{1}, \ldots, \lambda_{r} \in k^{\times}$and $\xi \in K^{\times}$satisfying (5.1). The map $f: \mathcal{T} \rightarrow X^{\mathrm{sm}}$ is defined on $\mathcal{T}_{U}$ by $\left(\mathbf{t}, \mathbf{z}_{1}, \ldots, \mathbf{z}_{r}\right) \mapsto\left(\mathbf{t}, \xi \mathbf{z}_{1}^{e_{1}} \cdots \mathbf{z}_{r}^{e_{r}}\right)$.

Proof. The proof proceeds along the lines of [22, Theorem 2.2] and [17, Proposition 2], based on the local description of universal torsors in [9, Theorem 2.3.1, Corollary 2.3.4]. Since $\mathcal{T}_{U}$ is defined over $\bar{k}$ by $u_{i, 1} \cdots u_{i, n}=\lambda_{i}^{-1} g_{i}(\mathbf{t}) \neq 0$ for $i=1, \ldots, r$, it is geometrically rational.

To prove strong approximation on the universal torsors as in Proposition 5.1 in the situation of Theorem 1.2, we use a generalization of the main analytic result [4, Theorem 5.2] of Browning and Matthiesen from linear forms to (not necessarily homogeneous) linear polynomials; see also [4, Remark 5.3]. To state the result, we introduce some notation.

Let $K$ be a number field of degree $n$, and let $N_{K / \mathbb{Q}}(\mathbf{z}) \in \mathbb{Z}\left[z_{1}, \ldots, z_{n}\right]$ be an associated norm form with integral coefficients. Let $f_{1}(\mathbf{t}), \ldots, f_{r}(\mathbf{t}) \in \mathbb{Z}\left[t_{1}, \ldots, t_{s}\right]$ be linear polynomials that are pairwise affinely independent over $\mathbb{Q}$, i.e., for $i \neq j \in\{1, \ldots, r\}$, the homogeneous parts $f_{i}(\mathbf{t})-f_{i}(0)$ and $f_{j}(\mathbf{t})-f_{j}(0)$ are not proportional over $\mathbb{Q}$. Consider the system of equations

$$
N_{K / \mathbb{Q}}\left(\mathbf{z}_{i}\right)=f_{i}(\mathbf{t}) \quad(i=1, \ldots, r) .
$$

Let $\mathfrak{D}_{+} \subset \mathbb{R}^{n}$ be the fundamental domain for the action of the free part of the norm-1subgroup of the unit group of $K$ as in [4, equation (2.3)]. Let $\Omega \subset[-1,1]^{s} \subset \mathbb{R}^{s}$ be a convex bounded set with $\left|f_{i}(T \Omega)\right| \leq T$, for $1 \leq i \leq r$ and sufficiently large $T \in \mathbb{R}$.

From [4, Definition 5.1], recall the definition of the representation function

$$
R\left(m ; \mathfrak{D}_{+}, \mathbf{z}^{\prime}, M\right):=\mathbf{1}_{m \neq 0} \cdot \#\left\{\mathbf{z}^{\prime \prime} \in \mathbb{Z}^{n} \cap \mathfrak{D}_{+}: N_{K / \mathbb{Q}}\left(\mathbf{z}^{\prime \prime}\right)=m, \mathbf{z}^{\prime \prime} \equiv \mathbf{z}^{\prime}(\bmod M)\right\},
$$

for $m \in \mathbb{Z}, M \in \mathbb{Z}_{>0}$ and $\mathbf{z}^{\prime} \in \mathbb{Z}^{n}$. 
Let $q^{\prime}=\left(\mathbf{t}^{\prime}, \mathbf{z}_{1}^{\prime}, \ldots, \mathbf{z}_{r}^{\prime}\right) \in \mathbb{Z}^{r n+s}$ and $M \in \mathbb{Z}_{>0}$. Then

$$
N(T):=\sum_{\substack{\mathbf{t}^{\prime \prime} \in \mathbb{Z}^{s} \cap T \Omega \\ \mathbf{t}^{\prime \prime} \equiv \mathbf{t}^{\prime}(\bmod M)}} \prod_{i=1}^{r} R\left(f_{i}\left(\mathbf{t}^{\prime \prime}\right) ; \mathfrak{D}_{+}, \mathbf{z}_{i}^{\prime}, M\right)
$$

is the number of solutions $q^{\prime \prime}=\left(\mathbf{t}^{\prime \prime}, \mathbf{z}_{1}^{\prime \prime}, \ldots, \mathbf{z}_{r}^{\prime \prime}\right) \in \mathbb{Z}^{r n+s}$ of (5.2) lying in $T \Omega \times\left(\mathfrak{D}_{+}\right)^{r}$ with $q^{\prime \prime} \equiv q^{\prime}(\bmod M)$ and $f_{1}\left(\mathbf{t}^{\prime \prime}\right) \cdots f_{r}\left(\mathbf{t}^{\prime \prime}\right) \neq 0$.

Let $r_{1}$ resp. $r_{2}$ be the number of real resp. complex places of $K$, let $D_{K}$ be its discriminant, and let $R_{K}^{(+)}$be its modified regulator as in [4, Remark 5.4]. Let

$$
c_{K}:=\frac{2^{r_{1}-1}(2 \pi)^{r_{2}} R_{K}^{(+)}}{\sqrt{\left|D_{K}\right|}} \text {. }
$$

Theorem 5.2. Let $f_{1}, \ldots, f_{r} \in \mathbb{Z}\left[t_{1}, \ldots, t_{S}\right]$ be linear polynomials that are pairwise affinely independent, let $K$ be a number field, let $M \in \mathbb{Z}$, and let $q^{\prime}=\left(\mathbf{t}^{\prime}, \mathbf{z}_{1}^{\prime}, \ldots, \mathbf{z}_{r}^{\prime}\right) \in \mathbb{Z}^{r n+s}$. Let $\epsilon$ be an arbitrary element of $\{ \pm\}^{r}$ if $K$ is not totally imaginary, and let $\epsilon=(+, \ldots,+)$ if $K$ is totally imaginary. Let $\Omega \subset[-1,1]^{s}$ be a convex bounded set whose closure is contained in

$$
\mathfrak{Q}_{\epsilon}:=\left\{\mathbf{t} \in \mathbb{R}^{s}: 0<\epsilon_{i}\left(f_{i}(\mathbf{t})-f_{i}(0)\right)<1 \text { for } i=1, \ldots, r\right\} .
$$

For $N(T)$ as in (5.3), we have

$$
N(T)=\beta_{\infty} \prod_{p} \beta_{p} \cdot T^{s}+o\left(T^{s}\right) \quad(T \rightarrow \infty)
$$

where

$$
\beta_{\infty}:=c_{K}^{r} \operatorname{vol}(\Omega)
$$

and $\prod_{p} \beta_{p}$ is absolutely convergent, with

$$
\beta_{p}:=\lim _{m \rightarrow \infty} \frac{1}{p^{m s}} \sum_{\substack{\mathbf{t}^{\prime \prime \prime} \in\left(\mathbb{Z} / p^{m} \mathbb{Z}\right)^{s} \\ \mathbf{t}^{\prime \prime \prime} \equiv \mathbf{t}^{\prime}\left(\bmod p^{v_{p}(M)}\right)}} \prod_{i=1}^{r} \frac{\rho\left(p^{m}, f_{i}\left(\mathbf{t}^{\prime \prime \prime}\right), \mathbf{z}_{i}^{\prime} ; p^{v_{p}(M)}\right)}{p^{m(n-1)}}
$$

and

$$
\begin{gathered}
\rho\left(p^{m}, A, \mathbf{z}^{\prime} ; p^{v_{p}(M)}\right):=\#\left\{\mathbf{z}^{\prime \prime \prime} \in\left(\mathbb{Z} / p^{m} \mathbb{Z}\right)^{n}: N_{K / \mathbb{Q}}\left(\mathbf{z}^{\prime \prime \prime}\right) \equiv A\left(\bmod p^{m}\right),\right. \\
\left.\mathbf{z}^{\prime \prime \prime} \equiv \mathbf{z}^{\prime}\left(\bmod p^{v_{p}(M)}\right)\right\}
\end{gathered}
$$

for $m \geq v_{p}(M)$ and $A \in \mathbb{Z} / p^{m} \mathbb{Z}$.

Proof. The proof follows [4, Sections 6-10]. We note that our condition on $\Omega$ implies that

$$
0<\epsilon_{i} f_{i}(T \Omega)<T
$$

for $i=1, \ldots, r$ and sufficiently large $T$. This allows us to apply [4, Proposition 7.10] as in [4, Proposition 8.2], but without decomposing $\Omega$ (in the inhomogeneous case, a decomposition that allows a direct application of [4, Proposition 7.10] does not seem to exist).

Proposition 5.3. Let $s \geq 2$. Let $Y \subset \mathbb{A}_{\mathbb{Q}}^{r n+s}$ be the variety defined by

$$
N_{K / \mathbb{Q}}\left(\mathbf{z}_{i}\right)=\lambda_{i}^{-1} g_{i}(\mathbf{t}) \quad(i=1, \ldots, r)
$$


with

$$
g_{i}\left(t_{1}, \ldots, t_{s}\right) \in \mathbb{Q}\left[t_{1}, \ldots, t_{s}\right]
$$

pairwise affinely independent linear polynomials and $\lambda_{i} \in \mathbb{Q}^{\times}$. If $K$ is not totally imaginary or if the polytope

$$
Q:=\left\{\mathbf{t} \in \mathbb{R}^{s}: \lambda_{i}^{-1} g_{i}(\mathbf{t})>0 \text { for } i=1, \ldots, r\right\}
$$

contains balls of arbitrarily large radius, then smooth strong approximation holds for $Y$ off $\infty$.

Proof. We may assume that the norm form $N_{K / \mathbb{Q}} \in \mathbb{Z}\left[z_{1}, \ldots, z_{n}\right]$ has integral coefficients. By rescaling $t_{1}, \ldots, t_{s}$ and $\mathbf{z}_{1}, \ldots, \mathbf{z}_{r}$, we may assume that

$$
\lambda_{i}^{-1} g_{i}(\mathbf{t}) \in \mathbb{Z}\left[t_{1}, \ldots, t_{s}\right] \quad(i=1, \ldots, r)
$$

are linear forms with integral coefficients. We define the integral model $\mathfrak{Y} \subset \mathbb{A}_{\mathbb{Z}}^{r n+s}$ of $Y$ by the same equations, but considered over $\mathbb{Z}$.

For smooth strong approximation on $Y$, we must show: for any finite set of places $S \subset \Omega_{\mathbb{Q}} \backslash\{\infty\}$ and for any $\left(q_{v}\right)=\left(\mathbf{t}_{v}, \mathbf{z}_{1, v}, \ldots, \mathbf{z}_{r, v}\right) \in Y^{\mathrm{sm}}\left(\mathbb{A}_{\mathbb{Q}}\right)$ with $q_{v} \in \mathfrak{Y}\left(\mathbb{Z}_{v}\right)$ for all $v \notin S \cup\{\infty\}$, there is a $q \in Y^{\mathrm{sm}}(\mathbb{Q})$ with $q \in \mathfrak{Y}\left(\mathbb{Z}_{v}\right)$ for all $v \notin S \cup\{\infty\}$ and $q$ arbitrarily close to $q_{v}$ for all $v \in S$. This is the same as finding $q \in Y^{\mathrm{sm}}(\mathbb{Q}) \cap \mathfrak{Y}\left(\mathbb{Z}_{S}\right)$ arbitrarily close to $q_{v}$ for all $v \in S$.

By the implicit function theorem, we may assume that the given $\left(q_{v}\right)$ satisfies

$$
g_{1}\left(\mathbf{t}_{v}\right) \cdots g_{r}\left(\mathbf{t}_{v}\right) \neq 0
$$

for all $v \in \Omega_{\mathbb{Q}}$.

Let $C \in \mathbb{Z}$ with $C^{-1} \in \mathbb{Z}_{S}$ (i.e., all prime factors of $C$ lie in $S$ ) be such that

$$
q_{v}^{\prime}=\left(\mathbf{t}_{v}^{\prime}, \mathbf{z}_{1, v}^{\prime}, \ldots, \mathbf{z}_{r, v}^{\prime}\right):=\left(C \mathbf{t}_{v}, C \mathbf{z}_{1, v}, \ldots, C \mathbf{z}_{r, v}\right) \in \mathbb{Z}_{v}^{r n+s}
$$

for all $v \in S$.

Let $Y^{\prime}$ be the variety obtained from $Y$ by the change of coordinates

$$
\left(\mathbf{t}, \mathbf{z}_{1}, \ldots, \mathbf{z}_{r}\right) \mapsto\left(C \mathbf{t}, C \mathbf{z}_{1}, \ldots, C \mathbf{z}_{r}\right) .
$$

An integral model $\mathfrak{Y}^{\prime}$ of $Y^{\prime}$ is given by

$$
N_{K / \mathbb{Q}}\left(\mathbf{z}_{i}\right)=f_{i}(\mathbf{t}) \quad(i=1, \ldots, r)
$$

with $f_{i}(\mathbf{t}):=C^{n} \lambda_{i}^{-1} g_{i}\left(C^{-1} \mathbf{t}\right) \in \mathbb{Z}[\mathbf{t}]$. This maps $\left(q_{v}\right) \in Y^{\mathrm{sm}}\left(\mathbb{A}_{\mathbb{Q}}\right)$ to $\left(q_{v}^{\prime}\right) \in Y^{\prime \mathrm{sm}}\left(\mathbb{A}_{\mathbb{Q}}\right)$ as above with $q_{v}^{\prime} \in \mathfrak{Y}^{\prime}\left(\mathbb{Z}_{v}\right)$ for all $v \in S$ (and of course still $q_{v}^{\prime} \in \mathfrak{Y}^{\prime}\left(\mathbb{Z}_{v}\right)$ for all $v \notin S \cup\{\infty\}$ ).

By strong approximation on $\mathbb{A}_{\mathbb{Z}}^{r n+s}$, we find $q^{\prime}=\left(\mathbf{t}^{\prime}, \mathbf{z}_{1}^{\prime}, \ldots, \mathbf{z}_{r}^{\prime}\right) \in \mathbb{Z}^{r n+s}$ arbitrarily close to $q_{v}$ for all $v \in S$.

Now we are looking for a point $q^{\prime \prime}=\left(\mathbf{t}^{\prime \prime}, \mathbf{z}_{1}^{\prime \prime}, \ldots, \mathbf{z}_{r}^{\prime \prime}\right) \in Y^{\prime \mathrm{sm}}(\mathbb{Q}) \cap \mathfrak{Y}^{\prime}(\mathbb{Z})$ very close to $q^{\prime}$ in the $v$-adic topology for all $v \in S$. This translates into the condition

$$
q^{\prime \prime} \equiv q^{\prime}(\bmod M)
$$

for a positive integer $M=\prod_{p \in S} p^{m^{\prime}}$ for some sufficiently large integer $m^{\prime}$. Once we have found such a $q^{\prime \prime} \in Y^{\prime \operatorname{sm}}(\mathbb{Q}) \cap \mathfrak{Y}^{\prime}(\mathbb{Z})$, then this is also very close to $q_{v}^{\prime} \in \mathfrak{Y}^{\prime}\left(\mathbb{Z}_{v}\right)$ for all $v \in S$, and then $q:=\left(C^{-1} \mathbf{t}^{\prime \prime}, C^{-1} \mathbf{z}_{1}^{\prime \prime}, \ldots, C^{-1} \mathbf{z}_{r}^{\prime \prime}\right) \in Y^{\mathrm{sm}}(\mathbb{Q}) \cap \mathfrak{Y}\left(\mathbb{Z}_{S}\right)$ is very close to $q v$ for all $v \in S$, completing the proof. 
By the definition of the number $N(T)$ in (5.3), where the condition $f_{1}\left(\mathbf{t}^{\prime \prime}\right) \cdots f_{r}\left(\mathbf{t}^{\prime \prime}\right) \neq 0$ ensures $q^{\prime \prime} \in Y^{\prime s m}(\mathbb{Q})$, it is enough to show $N(T)>0$ for some $T$.

There is an $\epsilon \in\{ \pm\}^{r}$ and a set $\Omega$ with positive volume satisfying the conditions of Theorem 5.2. Indeed, if $K$ is not totally imaginary, we choose $\epsilon \in\{ \pm\}^{r}$ such that

$$
Q_{\epsilon}:=\left\{\mathbf{t} \in \mathbb{R}^{s}: \epsilon_{i}\left(f_{i}(\mathbf{t})-f_{i}(0)\right)>0 \text { for } i=1, \ldots, r\right\}
$$

(which is a cone whose vertex is the origin) is non-empty; such an $\epsilon$ clearly exists. If $K$ is totally imaginary, we must choose $\epsilon=(+, \ldots,+)$. We note that our condition on $Q$ is equivalent to the condition that

$$
Q^{\prime}:=\left\{\mathbf{t} \in \mathbb{R}^{s}: f_{i}(\mathbf{t})>0 \text { for } i=1, \ldots, r\right\}
$$

contains balls of arbitrarily large radius. For $R \in \mathbb{R}_{>0}$, let $\mathbf{t}_{R}$ be the center of a ball of radius $R$ contained in $Q^{\prime}$. For sufficiently large $R$, we have $f_{i}\left(\mathbf{t}_{R}\right)>f_{i}(0)$, hence $\mathbf{t}_{R}$ lies in the cone $Q_{\epsilon}$ for $\epsilon=(+, \ldots,+)$, which is therefore non-empty. For any $K$, a point $\mathbf{t}$ in $Q_{\epsilon}$ sufficiently close to the origin satisfies $f_{i}(\mathbf{t})<1$ for $i=1, \ldots, r$, hence lies in $\mathfrak{Q}_{\epsilon}$ as in Theorem 5.2, and the same is true for a small enough closed ball $\Omega$ around $\mathbf{t}$.

Hence an application of Theorem 5.2 with this $\Omega$ gives $N(T)=\beta_{\infty} \prod_{p} \beta_{p} \cdot T^{s}+o\left(T^{s}\right)$ for large enough $T$, where $\beta_{\infty}=c_{K}^{r} \operatorname{vol}(\Omega)>0$.

Finally, we show that $\prod_{p} \beta_{p}>0$. By [4, Proposition 5.5] (which also holds for our inhomogeneous linear polynomials) there is an $L^{\prime}>0$ such that $\prod_{p>L^{\prime}} \beta_{p}>0$. Now we show that $\beta_{p}>0$ for any prime $v=p$. Let

$$
m^{\prime}:=2\left(v_{p}(M)+\max _{i=1, \ldots, r} v_{p}\left(f_{i}\left(\mathbf{t}_{p}^{\prime}\right)\right)+v_{p}(n)\right)+1 .
$$

Let $q^{\prime \prime \prime}=\left(\mathbf{t}^{\prime \prime \prime}, \mathbf{z}_{1}^{\prime \prime \prime}, \ldots, \mathbf{z}_{r}^{\prime \prime \prime}\right) \in \mathbb{Z}^{r n+s}$ be such that $q^{\prime \prime \prime} \equiv q_{p}^{\prime}\left(\bmod p^{m^{\prime}}\right)$. Then

$$
\rho\left(p^{m^{\prime}}, f_{i}\left(\mathbf{t}^{\prime \prime \prime}\right), \mathbf{z}_{i}^{\prime \prime \prime}, p^{v_{p}(M)}\right)>0 .
$$

Since $f_{i}\left(\mathbf{t}^{\prime \prime \prime}\right) \neq 0 \in \mathbb{Z} / p^{m^{\prime}} \mathbb{Z}$ because $f_{i}\left(\mathbf{t}_{p}^{\prime}\right) \neq 0 \in \mathbb{Z}_{p}$ by (5.6), we can apply [4, Lemma 3.4] to obtain

$$
\frac{\rho\left(p^{m}, f_{i}\left(\mathbf{t}^{\prime \prime \prime \prime}\right), \mathbf{z}_{i}^{\prime \prime \prime} ; p^{v_{p}(M)}\right)}{p^{m(n-1)}}=\frac{\rho\left(p^{m^{\prime}}, f_{i}\left(\mathbf{t}^{\prime \prime \prime}\right), \mathbf{z}_{i}^{\prime \prime \prime} ; p^{v_{p}(M)}\right)}{p^{m^{\prime}(n-1)}} \geq \frac{1}{p^{m^{\prime}(n-1)}}
$$

for all $m \geq m^{\prime}$ and all $\mathbf{t}^{\prime \prime \prime \prime} \in\left(\mathbb{Z} / p^{m} \mathbb{Z}\right)^{s}$ satisfying $\mathbf{t}^{\prime \prime \prime \prime} \equiv \mathbf{t}^{\prime \prime \prime}\left(\bmod p^{m^{\prime}}\right)$. Since $\mathbf{t}^{\prime}$ was chosen very close to $\mathbf{t}_{p}^{\prime}$ for $p \in S$, and since $v_{p}(M)=0$ for $p \notin S \cup\{\infty\}$, we note that

$$
\mathbf{t}^{\prime \prime \prime} \equiv \mathbf{t}_{p}^{\prime}\left(\bmod p^{m^{\prime}}\right)
$$

implies

$$
\mathbf{t}^{\prime \prime \prime} \equiv \mathbf{t}^{\prime}\left(\bmod p^{v_{p}(M)}\right) .
$$

Therefore, the $m$-th approximation $\beta_{p, m}$ of $\beta_{p}$ has at least $p^{\left(m-m^{\prime}\right) s}$ summands from these $\mathbf{t}^{\prime \prime \prime \prime}$ that are at least as large as $p^{-m s} \cdot\left(p^{-m^{\prime}(n-1)}\right)^{r}$, hence $\beta_{p, m} \geq p^{-m^{\prime}(s+r(n-1))}$. Therefore, $\beta_{p}>0$ for all primes $p$.

The complement of the hyperplanes $\left\{g_{1}(\mathbf{t})=0\right\}, \ldots,\left\{g_{r}(\mathbf{t})=0\right\}$ in affine $s$-space consists of open polytopes. The following lemma shows that essentially every other such polytope occurs as the interior of a connected component of $p\left(X^{\mathrm{sm}}(\mathbb{R})\right)$ if $K$ is totally imaginary. 
Lemma 5.4. Let $X$ be as in Theorem 1.2, with $P(\mathbf{t})=c g_{1}(\mathbf{t})^{e_{1}} \cdots g_{r}(\mathbf{t})^{e_{r}}$ as in Proposition 5.1 , for $k=\mathbb{Q}$. If $K$ is not totally imaginary, then $p\left(X^{\mathrm{sm}}(\mathbb{R})\right)=\mathbb{R}^{s}$. If $K$ is totally imaginary, then the connected components of $p\left(X^{\mathrm{sm}}(\mathbb{R})\right)$ are precisely the sets

$$
\begin{aligned}
Q_{\epsilon}:=\left\{\mathbf{t} \in \mathbb{R}^{s}:\right. & g_{i}(\mathbf{t})=0 \text { for at most one } i \in\{1, \ldots, r\} \text { with } e_{i}=1, \\
& \left.\epsilon_{i} g_{i}(\mathbf{t})>0 \text { for all other } i \in\{1, \ldots, r\}\right\}
\end{aligned}
$$

for all $\epsilon=\left(\epsilon_{1}, \ldots, \epsilon_{r}\right) \in\{ \pm\}^{r}$ such that $\left(\epsilon_{1}\right)^{e_{1}} \cdots\left(\epsilon_{r}\right)^{e_{r}} \cdot c>0$.

Proof. Since $\bar{X}$ is defined by $c g_{1}(\mathbf{t})^{e_{1}} \cdots g_{r}(\mathbf{t})^{e_{r}}=u_{1} \cdots u_{n}$, the singular locus of $\bar{X}$ is the union of all $\left\{g_{i}(\mathbf{t})=g_{j}(\mathbf{t})=u_{l}=u_{m}=0\right\}$ for $l \neq m \in\{1, \ldots, n\}$ and $i, j \in\{1, \ldots, r\}$ with either $i=j$ and $e_{i}>1$ or $i \neq j$.

If $K$ is not totally imaginary, the norm form $N_{K / \mathbb{Q}}$ is indefinite over $\mathbb{R}$, and for any $\mathbf{t} \in \mathbb{R}^{s}$, the number $P(\mathbf{t})$ can be represented by $N_{K / \mathbb{Q}}(\mathbf{z})$ for some $\mathbf{z} \in \mathbb{R}^{n}$ with $(\mathbf{t}, \mathbf{z}) \in X^{\mathrm{sm}}(\mathbb{R})$.

If $K$ is totally imaginary, the norm form $N_{K / \mathbb{Q}}$ is positive definite over $\mathbb{R}$, hence for any $(\mathbf{t}, \mathbf{z}) \in X(\mathbb{R})$ with $P(\mathbf{t})=0$, we have $\mathbf{z}=0$, hence $\mathbf{t} \in p\left(X^{\mathrm{sm}}(\mathbb{R})\right)$ if and only if $g_{i}(\mathbf{t})=0$ for at most one $i \in\{1, \ldots, r\}$ that satisfies additionally $e_{i}=1$. This clearly splits into the connected components described in the statement of the lemma.

Proof of Theorem 1.2. We want to apply Lemma 3.1. Let $U:=X \cap\{P(\mathbf{t}) \neq 0\}$. Let $f: \mathcal{T} \rightarrow X^{\mathrm{sm}}$ be a universal torsor, with local description $\mathcal{T}_{U} \rightarrow U$ as in Proposition 5.1 with associated $\lambda_{1}, \ldots, \lambda_{r} \in \mathbb{Q}^{\times}$and $\xi \in K^{\times}$. Also by Proposition 5.1, $\mathcal{T}_{U}$ is geometrically rational, hence geometrically integral.

Let $Y \subset \mathbb{A}_{\mathbb{Q}}^{r n+s}$ be defined by

$$
N_{K / \mathbb{Q}}\left(\mathbf{z}_{i}\right)=\lambda_{i}^{-1} g_{i}(\mathbf{t}) \quad(i=1, \ldots, r) .
$$

By Proposition 5.1, we have an open immersion $\mathcal{T}_{U} \subset Y$.

The morphism $g: Y \rightarrow X$ defined by $\left(\mathbf{t}, \mathbf{z}_{1}, \ldots, \mathbf{z}_{r}\right) \mapsto\left(\mathbf{t}, \xi \mathbf{z}_{1}^{e_{1}} \cdots \mathbf{z}_{r}^{e_{r}}\right)$ clearly agrees on $\mathcal{T}_{U}$ with $f: \mathcal{T} \rightarrow X^{\text {sm }}$.

If $K$ is not totally imaginary, every such $Y$ satisfies smooth strong approximation off $\infty$ by Proposition 5.3, hence Lemma 3.1 implies Theorem 1.2. Furthermore,

$$
C=p\left(X^{\mathrm{sm}}(\mathbb{R})\right)=\mathbb{R}^{s}
$$

by Lemma 5.4 .

If $K$ is totally imaginary, Proposition 5.3 gives smooth strong approximation off $\infty$ not necessarily for all $Y$. Following the proof of Lemma 3.1, we see that $X^{\mathrm{sm}}(k)$ is dense in the image of $V^{\operatorname{Br}\left(X^{\mathrm{sm}}\right)}$ in $X\left(\mathbb{A}_{k}^{f}\right)$, where $V$ is the set of all $\left(p_{v}\right) \in X^{\mathrm{sm}}\left(\mathbb{A}_{k}\right)$ that can be lifted to $\left(r_{v}\right) \in \mathcal{T}\left(\mathbb{A}_{k}\right)$ for a universal torsor $f: \mathcal{T} \rightarrow X^{\text {sm }}$ such that the associated $Y$ satisfies strong approximation off $\infty$.

To complete the proof of Theorem 1.2, it remains to show that $p^{-1}(C) \times X^{\mathrm{sm}}\left(\mathbb{A}_{k}^{f}\right) \subset V$. Indeed, consider $\left(p_{v}\right)=\left(\mathbf{t}_{v}, \mathbf{z}_{v}\right) \in X^{\mathrm{sm}}\left(\mathbb{A}_{k}\right)$ with $\mathbf{t}_{\infty}$ in an unbounded connected component $Q_{\epsilon}$ of $C$ for some $\epsilon \in\{ \pm\}^{r}$ as in Lemma 5.4. Then we have its lift $\left(r_{v}\right) \in \mathcal{T}\left(\mathbb{A}_{k}\right)$ for some universal torsor $f: \mathcal{T} \rightarrow X^{\mathrm{sm}}$, and very close to it $\left(r_{v}^{\prime}\right)=\left(\mathbf{t}_{v}^{\prime}, \mathbf{z}_{1, v}^{\prime}, \ldots, \mathbf{z}_{r, v}^{\prime}\right) \in \mathcal{T}_{U}\left(\mathbb{A}_{k}\right)$. By choosing $\left(r_{v}^{\prime}\right)$ close enough to $\left(r_{v}\right)$, we know that $\left(f\left(r_{v}^{\prime}\right)\right)=\left(\mathbf{t}_{v}^{\prime}, \xi \mathbf{z}_{1, v}^{\prime e_{1}} \cdots \mathbf{z}_{r, v}^{\prime e_{r}}\right) \in U\left(\mathbb{A}_{k}\right)$ has $\mathbf{t}_{\infty}^{\prime}$ on the same component $Q_{\epsilon}$ as $\mathbf{t}_{\infty}$. Since $P\left(\mathbf{t}_{\infty}^{\prime}\right) \neq 0$, we have $\epsilon=\left(\epsilon_{1}, \ldots, \epsilon_{r}\right)$ with $\epsilon_{i}=\operatorname{sign}\left(g_{i}\left(\mathbf{t}_{\infty}^{\prime}\right)\right) \in\{ \pm\}$. Since $\left(r_{v}^{\prime}\right)$ satisfies the equations defining $\mathcal{T}_{U}$ as in Proposition 5.1, 
we have

$$
\lambda_{i}^{-1} g_{i}\left(\mathbf{t}_{\infty}^{\prime}\right)=N_{K / \mathbb{Q}}\left(\mathbf{z}_{i, \infty}^{\prime}\right)>0,
$$

hence $\operatorname{sign}\left(\lambda_{i}^{-1}\right)=\operatorname{sign}\left(g_{i}\left(\mathbf{t}_{\infty}^{\prime}\right)\right)=\epsilon_{i}$. Therefore, the associated $Q$ in Proposition 5.3 is precisely the interior of $Q_{\epsilon}$. Since $Q_{\epsilon}$ contains balls of arbitrarily large radius by assumption, the same holds for $Q$, and Proposition 5.3 tells us that $Y$ satisfies smooth strong approximation, hence $\left(p_{v}\right) \in V$ as required.

If $g_{1}(\mathbf{t}), \ldots, g_{r}(\mathbf{t})$ are linear forms, the conditions $\epsilon_{i} g_{i}(\mathbf{t})>0$ define open halfspaces in $\mathbb{R}^{s}$ bounded by the hyperplanes $\left\{g_{i}(\mathbf{t})=0\right\}$ through the origin. Therefore, the interior of every connected component of $p\left(X^{\mathrm{sm}}(\mathbb{R})\right)$ is a cone whose vertex is the origin, hence every connected component contains balls of arbitrarily large radius, and $C=p\left(X^{\mathrm{sm}}(\mathbb{R})\right)$.

Remark 5.5. Theorem 5.2 is restricted to $s \geq 2$ due to fundamental restrictions in the additive combinatorics techniques. Nevertheless, [4, Theorem 1.1] proves a weak approximation result also for $s=1$ by homogenizing the equations describing the vertical torsors (see [4, Section 1.2]), using that a smooth variety satisfies weak approximation if and only if the same holds for an open subset. The latter is not true for strong approximation, hence we can deduce Theorem 1.2 only for $s \geq 2$.

Remark 5.6. For $X$ as in Theorem 1.2, write $P(\mathbf{t})=c g_{1}(\mathbf{t})^{e_{1}} \cdots g_{r}(\mathbf{t})^{e_{r}}$ as in Proposition 5.1. Under the assumption that $\operatorname{gcd}\left(e_{1}, \ldots, e_{r}\right)=1$, the Picard group $\operatorname{Pic}\left(\bar{X}^{\mathrm{sm}}\right)$ is free of finite rank, hence $\operatorname{Br}_{1}\left(X^{\mathrm{sm}}\right) / \operatorname{Br}_{0}\left(X^{\mathrm{sm}}\right) \cong H^{1}\left(k, \operatorname{Pic}\left(\bar{X}^{\mathrm{sm}}\right)\right.$ is finite.

Therefore by Remark 2.4, $X$ also satisfies central strong approximation with algebraic Brauer-Manin obstruction off $\infty$ under the assumption that $K$ is not totally imaginary or that $g_{1}(\mathbf{t}), \ldots, g_{r}(\mathbf{t})$ are linear forms.

By Remark 2.2, Theorem 1.2 implies that $X$ also satisfies the smooth integral Hasse principle (in the sense of Definition 2.1). This can be stated explicitly as follows.

Corollary 5.7. Let $P(\mathbf{t}) \in \mathbb{Z}\left[t_{1}, \ldots, t_{s}\right]$ be a product of linear polynomials over $\mathbb{Z}$ that are over $\mathbb{Q}$ pairwise proportional or affinely independent. Let $K / \mathbb{Q}$ be an extension of number fields of degree $n$, and let $N_{K / \mathbb{Q}}(\mathbf{z}) \in \mathbb{Z}\left[z_{1}, \ldots, z_{n}\right]$ be an associated norm form with integral coefficients. Suppose that $K$ is not totally imaginary or that the factors of $P(\mathbf{t})$ are linear forms. Let $\mathfrak{X} \subset \mathbb{A}_{\mathbb{Z}}^{n+s}$ be the affine scheme defined by

$$
P(\mathbf{t})=N_{K / \mathbb{Q}}(\mathbf{z}) .
$$

Let $X=\mathfrak{X}_{\mathbb{Q}}$ be the generic fiber. If there are $q_{v}=\left(\mathbf{t}_{v}, \mathbf{z}_{v}\right) \in \mathfrak{X}\left(\mathbb{Z}_{v}\right)$ for all finite places $v \in \Omega_{\mathbb{Q}}$ and $q_{\infty}=\left(\mathbf{t}_{\infty}, \mathbf{z}_{\infty}\right) \in X(\mathbb{R})$ such that $\left(q_{v}\right)_{v \in \Omega_{\mathbb{Q}}}$ is orthogonal to $\operatorname{Br}_{1}\left(X^{\mathrm{sm}}\right)$, with $P\left(\mathbf{t}_{v}\right) \in \mathbb{Z}_{v}^{\times}$ for almost all $v<\infty_{k}$ and $P\left(\mathbf{t}_{v}\right) \in k_{v}^{\times}$for all other $v \in \Omega_{\mathbb{Q}}$, then there are integral points on $\mathfrak{X}$.

Proof. The conditions on $P\left(\mathbf{t}_{v}\right)$ ensure that

$$
\left(q_{v}\right) \in U\left(\mathbb{A}_{k}\right) \subset X^{\mathrm{sm}}\left(\mathbb{A}_{k}\right),
$$

where $U:=X \cap\{P(\mathbf{t}) \neq 0\} \subset X^{\mathrm{sm}}$. Hence $\left(q_{v}\right)$ lies in the set (2.1). As in Remark 2.2, we obtain an integral point on $\mathfrak{X}$. 


\section{A counterexample}

Now we consider the case that $[K: k]=2$ and $P(t)$ is the product of pairwise distinct linear factors.

Proposition 6.1. Let $k$ be a field of characteristic 0 with $H^{3}\left(k, \bar{k}^{\times}\right)=0$. Furthermore, let $K:=k(\sqrt{d})$ be a quadratic extension of $k$. Let $P(t)=c\left(t-a_{1}\right) \cdots\left(t-a_{r}\right) \in k[t]$ with pairwise distinct $a_{1}, \ldots, a_{r} \in k$. Let $X$ be defined by (1.1) with $s=1$. Then

$$
\operatorname{Br}(X) / \operatorname{Br}_{0}(X) \cong(\mathbb{Z} / 2 \mathbb{Z})^{r-1}
$$

is generated by the classes of the quaternion algebras $\left(t-a_{i}, d\right) \in \operatorname{Br}(X)$ for $i=1, \ldots, r-1$.

Proof. Let $U$ be defined again by $P(t) \neq 0$. Analogously to (4.8), we have the exact sequence

$$
0 \rightarrow \mathbb{Z} \rightarrow \mathbb{Z}^{r} \oplus \mathbb{Z}[K / k] \rightarrow \widehat{T} \rightarrow 0,
$$

hence $\widehat{T} \cong \mathbb{Z}^{r-1} \oplus \mathbb{Z}[K / k]$ as a $\Gamma_{k}$-module. Furthermore, we have $\widehat{M} \cong \mathbb{Z}[K / k]^{r}$.

We have

$$
H^{1}(k, \operatorname{Pic}(\bar{X})) \cong H^{1}\left(K / k, \operatorname{Pic}\left(X_{K}\right)\right) \cong H^{2}\left(K / k, K[U]^{\times} / K^{\times}\right) \cong(\mathbb{Z} / 2 \mathbb{Z})^{r-1} .
$$

Indeed, for the first isomorphism, we note that $\operatorname{Pic}(\bar{X})$ is torsion-free and split by $K$, for the second isomorphism, we use (4.4) and $H^{i}(K / k, \widehat{M})=0$ for $i>0$ since $\hat{M} \cong \mathbb{Z}[K / k]^{r}$ is an induced $\operatorname{Gal}(K / k)$-module, and for the third isomorphism, we note that $K[U]^{\times} / K^{\times}$is $\mathbb{Z}^{r-1} \oplus \mathbb{Z}[K / k]$ as a $\operatorname{Gal}(K / k)$-module, as in our computation of $\widehat{T}$ above.

Since $X$ is smooth, Lemma 4.3 implies $\operatorname{Br}(X) / \operatorname{Br}_{0}(X) \cong(\mathbb{Z} / 2 \mathbb{Z})^{r-1}$.

Now we describe $\operatorname{Br}(X) / \operatorname{Br}_{0}(X)$ explicitly. Let $\beta_{i}$ be the quaternion algebra $\left(t-a_{i}, d\right)$ over $k(X)$.

First we show that $\beta_{i} \in \operatorname{Br}(X)$. Indeed, the quaternion algebra $\left(t-a_{i}, d\right)$ is clearly welldefined on $U_{i}=\left\{t \neq a_{i}\right\} \subset X$, and $\left(P(t) /\left(t-a_{i}\right), d\right)$ is well-defined on $U_{i}^{\prime}=\bigcap_{j \neq i}\left\{t \neq a_{j}\right\}$. Since $U_{i} \cup U_{i}^{\prime}=X$ and $\left(t-a_{i}, d\right)=\left(P(t) /\left(t-a_{i}\right), d\right)$ in $\operatorname{Br}(k(X))$ (since $P(t)=N_{K / k}(\mathbf{z})$ implies $\left.(P(t), d)=\left(N_{K / k}(\mathbf{z}), d\right)=0\right)$, we can extend $\left(t-a_{i}, d\right)$ to a well-defined element of $\operatorname{Br}(X)$.

Next we show that the quaternion algebras $\beta_{1}, \ldots, \beta_{r-1}$ are $\mathbb{Z} / 2 \mathbb{Z}$-linearly independent elements in $\operatorname{Br}(k(X)) / \operatorname{Br}_{0}(X)$. Again let $p: X \rightarrow \mathbb{A}_{k}^{1}$ be the projection to the $t$-coordinate. This induces a map $\operatorname{Br}(k(t)) \rightarrow \operatorname{Br}(k(X))$. Let $\eta$ be the generic point of $\mathbb{A}_{k}^{1}$ and $X_{\eta}$ the generic fiber of $p$. By [34, Satz, p. 465], the kernel of $\operatorname{Br}(k(t)) \rightarrow \operatorname{Br}(k(X))$ is generated by $(P(t), d)$, hence the induced map $\phi: \operatorname{Br}(k(t)) \rightarrow \operatorname{Br}(k(X)) / \operatorname{Br}_{0}(X)$ has kernel generated by $(P(t), d)$ and $\operatorname{Br}(k)$. For $n_{1}, \ldots, n_{r-1} \in \mathbb{Z} / 2 \mathbb{Z}$, let $\beta:=\sum_{i=1}^{r-1} n_{i} \beta_{i} \in \operatorname{Br}(k(t))$. We claim that $\beta \in \operatorname{ker} \phi$ if and only if $\beta=0$, which would imply the linear independence of $\beta_{1}, \ldots, \beta_{r-1}$ and complete the proof of this theorem.

Indeed, let $\chi$ be the non-trivial character of $\operatorname{Gal}(K / k)$, and let $D_{i}$ be the divisor $\left\{t=a_{i}\right\}$ of $\mathbb{A}_{k}^{1}$, for $i=1, \ldots, r$. By [12, Proposition 1.1.3], the residue of $\beta_{i}$ on $D_{i}$ is $\chi$, and the residue of $\beta_{i}$ on any other divisor of $\mathbb{A}_{k}^{1}$ is 0 . Hence the residue of $(P(t), d)$ is $\chi$ on $D_{1}, \ldots, D_{r}$ and 0 on any other divisor, and any element of $\operatorname{ker} \phi$ has the same residue $(\chi$ or 0$)$ on all of $D_{1}, \ldots, D_{r}$. But since $\beta$ has residue 0 on $D_{r}$, it must have residue 0 on $D_{1}, \ldots, D_{r-1}$ as well, which is only possible for $n_{1}=\cdots=n_{r-1}=0$, i.e., $\beta=0$. 
The following example illustrates that in Question 1.3, an unboundedness condition at an archimedean place is necessary. We construct a variety over $\mathbb{Q}$ with one bounded and one unbounded connected component over $\mathbb{R}$ and a point orthogonal to the Brauer group lying on the bounded component that cannot be approximated arbitrarily close. In particular, it is not enough to require that $X\left(k_{v}\right)$ is unbounded for one archimedean $v$.

Example 6.2. Let $X \subset \mathbb{A}_{\mathbb{Q}}^{3}$ be defined by

$$
t(t-2)(t-10)=x^{2}+y^{2} .
$$

We note that the projection $p: X \rightarrow \mathbb{A}_{\mathbb{Q}}^{1}$ to the $t$-coordinate has the bounded connected component $[0,2]$ and the unbounded connected component $[10, \infty)$. In fact, $X(\mathbb{R})$ has precisely two connected components, namely $X_{1}:=p^{-1}([0,2])$ bounded, $X_{2}:=p^{-1}([10, \infty))$ unbounded.

Our variety $X$ has an adelic point $\left(q_{v}\right) \in X(\mathbb{R}) \times \prod_{p} X\left(\mathbb{Z}_{p}\right)$ given by

$$
q_{v}=\left(t_{v}, x_{v}, y_{v}\right):= \begin{cases}\left(5, x_{5}, y_{5}\right), & v=5, \\ (1,3,0), & v \neq 5,\end{cases}
$$

where $\left(x_{5}, y_{5}\right) \in \mathbb{Z}_{5}^{2}$ is a solution of $x_{5}^{2}+y_{5}^{2}=-75$, which exists by Hensel's lemma.

This point is orthogonal to $\operatorname{Br}(X)$. Indeed, $\operatorname{Br}(X) / \operatorname{Br}_{0}(X)$ is generated by $\beta_{1}:=(t,-1)$ and $\beta_{2}:=(t-2,-1)$ by Proposition 6.1. We have

$$
\begin{aligned}
\sum_{v \in \Omega_{\mathbb{Q}}} \beta_{i}\left(q_{v}\right) & =\sum_{v \neq 5} \operatorname{inv}_{v}\left(\beta_{i}((1,3,0))\right)+\operatorname{inv}_{5}\left(\beta_{i}\left(\left(5, x_{5}, y_{5}\right)\right)\right) \\
& =\operatorname{inv}_{5}\left(\beta_{i}((1,3,0))\right)+\operatorname{inv}_{5}\left(\beta_{i}\left(\left(5, x_{5}, y_{5}\right)\right)\right)=0
\end{aligned}
$$

since $(1,-1)_{5}=(5,-1)_{5}=(-1,-1)_{5}=(3,-1)_{5}=0$.

Our goal is to show by contradiction that there is no integral point $q=(t, x, y) \in X(\mathbb{Z})$ that is very close to our adelic point $\left(q_{v}\right)$ at the places 2 and 5. More precisely, we show that $|t-1|_{2} \leq \frac{1}{8}$ and $|t-5|_{5} \leq \frac{1}{25}$ are impossible. This is done via the following claim.

Claim. Given $\left(q_{v}^{\prime}\right)=\left(t_{v}^{\prime}, x_{v}^{\prime}, y_{v}^{\prime}\right) \in\left(X(\mathbb{R}) \times \prod_{p} X\left(\mathbb{Z}_{p}\right)\right)^{\operatorname{Br}(X)}$ satisfying $\left|t_{2}^{\prime}-1\right|_{2} \leq \frac{1}{8}$, then $q_{\infty}^{\prime}$ lies in the bounded real component $X_{1}$.

This claim leads to our goal as follows. A point $q \in X(\mathbb{Z})$ very close to $\left(q_{v}\right)$ at 2 and 5 lies on $X_{1}$ by the claim. By the product formula, we get the contradiction

$$
1=\prod_{v \in \Omega_{k}}|t|_{v} \leq|t|_{\infty} \cdot|t|_{5} \leq 2 \cdot \frac{1}{5}<1,
$$

using $t \in \mathbb{Z} \backslash\{0\}$ and $|t-5|_{5} \leq \frac{1}{25}$. Hence such a $q \in X(\mathbb{Z})$ does not exist, and our adelic point $\left(q_{v}\right)$ cannot be approximated closely. we claim

It remains to prove the claim. For finite $p \neq 2,5$ and any $q_{p}^{\prime}=\left(t_{p}^{\prime}, x_{p}^{\prime}, y_{p}^{\prime}\right) \in X\left(\mathbb{Z}_{p}\right)$,

$$
\beta_{i}\left(q_{p}\right)=0 \text { in } \operatorname{Br}\left(\mathbb{Q}_{p}\right) \text { for } i=1,2 .
$$

If the Legendre symbol $\left(\frac{-1}{p}\right)=1$, then $\left(t_{p}^{\prime},-1\right)=0$ in $\operatorname{Br}\left(\mathbb{Q}_{p}\right)$ for any $t_{p}^{\prime} \in \mathbb{Q}_{p}^{\times}$. Therefore, 
we may assume $\left(\frac{-1}{p}\right)=-1$, hence $\mathbb{Q}_{p}(\sqrt{-1}) / \mathbb{Q}_{p}$ is unramified of degree 2 , so $x_{p}^{\prime 2}+y_{p}^{\prime 2}$ has even valuation at $p$. If $v_{p}\left(t_{p}^{\prime}\right)=0$, then $\left(t_{p}^{\prime},-1\right)=0$ in $\operatorname{Br}\left(\mathbb{Q}_{p}\right)$. If $v_{p}\left(t_{p}^{\prime}\right) \geq 1$, then

$$
v_{p}\left(t_{p}^{\prime}-2\right)=v_{p}\left(t_{p}^{\prime}-10\right)=0 ;
$$

because of $(6.1), v_{p}\left(t_{p}^{\prime}\right)$ is also even, hence we have $\left(t_{p}^{\prime},-1\right)=0$ in $\operatorname{Br}\left(\mathbb{Q}_{p}\right)$. Similarly, we prove $\left(t_{p}^{\prime}-2,-1\right)=0$.

For any $\left(q_{v}^{\prime}\right)=\left(t_{v}^{\prime}, x_{v}^{\prime}, y_{v}^{\prime}\right) \in\left(X(\mathbb{R}) \times \prod_{p} X\left(\mathbb{Z}_{p}\right)\right)^{\operatorname{Br}(X)}$ with $|t-1|_{2} \leq \frac{1}{8}$, we obviously have $t \neq 0$. By (6.2),

$$
\begin{aligned}
\sum_{v \in \Omega_{k}} \operatorname{inv}_{v}\left(\beta_{i}(q)\right) & =\operatorname{inv}_{2}\left(\beta_{i}(q)\right)+\operatorname{inv}_{5}\left(\beta_{i}(q)\right)+\operatorname{inv}_{\infty}\left(\beta_{i}(q)\right) \\
& = \begin{cases}(t,-1)_{\infty}, & i=1 \\
1+(t-2,-1)_{\infty}, & i=2\end{cases}
\end{aligned}
$$

since $(t,-1)_{5}=(t-2,-1)_{5}=0$ (because $\left.\left(\frac{-1}{5}\right)=1\right)$ and since $(t,-1)_{2}=(1,-1)_{2}=0$ and $(t-2,-1)=(1-2,-1)_{2}=1$ (because $\left.|t-1|_{2} \leq \frac{1}{8}\right)$.

By global class field theory,

$$
\sum_{v \in \Omega_{k}} \operatorname{inv}_{v}\left(\beta_{i}(q)\right)=0
$$

hence $(t,-1)_{\infty}=0$ and $(t-2,-1)_{\infty}=1$. Therefore, $0 \leq t \leq 2$, hence $q_{\infty}^{\prime} \in X_{1}$, which was our claim.

\section{References}

[1] M. Borovoi, The Brauer-Manin obstructions for homogeneous spaces with connected or abelian stabilizer, J. reine angew. Math. 473 (1996), 181-194.

[2] M. Borovoi and C. Demarche, Manin obstruction to strong approximation for homogeneous spaces, Comment. Math. Helv. 88 (2013), no. 1, 1-54.

[3] T.D. Browning and D. R. Heath-Brown, Quadratic polynomials represented by norm forms, Geom. Funct. Anal. 22 (2012), no. 5, 1124-1190.

[4] T.D. Browning and L. Matthiesen, Norm forms for arbitrary number fields as products of linear polynomials, preprint 2013, http://arxiv.org/abs/1307.7641.

[5] T. D. Browning, L. Matthiesen and A. N. Skorobogatov, Rational points on pencils of conics and quadrics with many degenerate fibers, Ann. of Math. (2) 180 (2014), no. 1, 381-402.

[6] J.-L. Colliot-Thélène, Points rationnels sur les fibrations, in: Higher dimensional varieties and rational points (Budapest 2001), Bolyai Soc. Math. Stud. 12, Springer, Berlin (2003), 171-221.

[7] J.-L. Colliot-Thélène, D. Coray and J.-J. Sansuc, Descente et principe de Hasse pour certaines variétés rationnelles, J. reine angew. Math. 320 (1980), 150-191.

[8] J.-L. Colliot-Thélène and D. Harari, Approximation forte en famille, J. reine angew. Math. (2013), DOI 10.1515/crelle-2013-0092.

[9] J.-L. Colliot-Thélène and J.-J. Sansuc, La descente sur les variétés rationnelles. II, Duke Math. J. 54 (1987), no. 2, 375-492.

[10] J.-L. Colliot-Thélène, J.-J. Sansuc and P. Swinnerton-Dyer, Intersections of two quadrics and Châtelet surfaces. I, J. reine angew. Math. 373 (1987), 37-107.

[11] J.-L. Colliot-Thélène, J.-J. Sansuc and P. Swinnerton-Dyer, Intersections of two quadrics and Châtelet surfaces. II, J. reine angew. Math. 374 (1987), 72-168.

[12] J.-L. Colliot-Thélène and P. Swinnerton-Dyer, Hasse principle and weak approximation for pencils of SeveriBrauer and similar varieties, J. reine angew. Math. 453 (1994), 49-112.

[13] J.-L. Colliot-Thélène and O. Wittenberg, Groupe de Brauer et points entiers de deux familles de surfaces cubiques affines, Amer. J. Math. 134 (2012), no. 5, 1303-1327. 
[14] J.-L. Colliot-Thélène and F. Xu, Brauer-Manin obstruction for integral points of homogeneous spaces and representation by integral quadratic forms, Compos. Math. 145 (2009), no. 2, 309-363.

[15] J.-L. Colliot-Thélène and $F . X u$, Strong approximation for the total space of certain quadratic fibrations, Acta Arith. 157 (2013), no. 2, 169-199.

[16] C. Demarche, Le défaut d'approximation forte dans les groupes linéaires connexes, Proc. Lond. Math. Soc. (3) 102 (2011), no. 3, 563-597.

[17] U. Derenthal, A. Smeets and D. Wei, Universal torsors and values of quadratic polynomials represented by norms, Math. Ann. 361 (2015), no. 3-4, 1021-1042.

[18] F. Gundlach, Integral Brauer-Manin obstructions for sums of two squares and a power, J. Lond. Math. Soc. (2) 88 (2013), no. 2, 599-618.

[19] D. Harari, Méthode des fibrations et obstruction de Manin, Duke Math. J. 75 (1994), no. 1, 221-260.

[20] D. Harari, Le défaut d'approximation forte pour les groupes algébriques commutatifs, Algebra Number Theory 2 (2008), no. 5, 595-611.

[21] Y. Harpaz, A. N. Skorobogatov and O. Wittenberg, The Hardy-Littlewood conjecture and rational points, Compos. Math. 150 (2014), no. 12, 2095-2111.

[22] D. R. Heath-Brown and A.N. Skorobogatov, Rational solutions of certain equations involving norms, Acta Math. 189 (2002), no. 2, 161-177.

[23] A. J. Irving, Cubic polynomials represented by norm forms, preprint 2013, http://arxiv.org/abs/1310. 6158.

[24] G. Karpilovsky, The Schur multiplier, London Math. Soc. Monogr. New Ser. 2, Clarendon Press, New York 1987.

[25] A. Kresch and Y. Tschinkel, Two examples of Brauer-Manin obstruction to integral points, Bull. Lond. Math. Soc. 40 (2008), no. 6, 995-1001.

[26] Y. I. Manin, Le groupe de Brauer-Grothendieck en géométrie diophantienne, in: Actes du congrès international des mathématiciens (Nice 1970), Tome 1, Gauthier-Villars, Paris (1971), 401-411.

[27] V. Platonov and A. Rapinchuk, Algebraic groups and number theory, Pure Appl. Math. 139, Academic Press, Boston 1994.

[28] D. Schindler and A. N. Skorobogatov, Norms as products of linear polynomials, J. Lond. Math. Soc. (2) 89 (2014), no. 2, 559-580.

[29] A. N. Skorobogatov, Beyond the Manin obstruction, Invent. Math. 135 (1999), no. 2, 399-424.

[30] D. Wei, On the equation $N_{K / k}(\Xi)=P(t)$, Proc. Lond. Math. Soc. (3) 109 (2014), no. 6, 1402-1434.

[31] D. Wei, Strong approximation for the variety containing a torus, preprint 2014, http://arxiv.org/abs/ 1403.1035.

[32] D. Wei and F. Xu, Integral points for multi-norm tori, Proc. Lond. Math. Soc. (3) 104 (2012), no. 5, 1019-1044.

[33] D. Wei and F. Xu, Integral points for groups of multiplicative type, Adv. Math. 232 (2013), 36-56.

[34] E. Witt, Über ein Gegenbeispiel zum Normensatz, Math. Z. 39 (1935), no. 1, 462-467.

Ulrich Derenthal, Institut für Algebra, Zahlentheorie und Diskrete Mathematik,

Leibniz Universität Hannover, Welfengarten 1, 30167 Hannover, Germany

e-mail: derenthal@math.uni-hannover.de

Dasheng Wei, Academy of Mathematics and System Science, CAS, Beijing 100190, P. R. China

e-mail: dshwei@amss.ac.cn

Eingegangen 19. August 2014, in revidierter Fassung 27. November 2014 